\title{
lmridge: A Comprehensive R Package for Ridge Regression
}

\author{
by Muhammad Imdad Ullah, Muhammad Aslam, and Saima Altaf
}

\begin{abstract}
The ridge regression estimator, one of the commonly used alternatives to the conventional ordinary least squares estimator, avoids the adverse effects in the situations when there exists some considerable degree of multicollinearity among the regressors. There are many software packages available for estimation of ridge regression coefficients. However, most of them display limited methods to estimate the ridge biasing parameters without testing procedures. Our developed package, lmridge can be used to estimate ridge coefficients considering a range of different existing biasing parameters, to test these coefficients with more than 25 ridge related statistics, and to present different graphical displays of these statistics.
\end{abstract}

\section{Introduction}

For data collected either from a designed experiment or from an observational study, the ordinary least squares (OLS) method does not provide precise estimates of the effect of any explanatory variable (regressor) when regressors are interdependent (collinear with each other). Consider a multiple linear regression (MLR) model,

$$
y=X \beta+\varepsilon,
$$

where $y$ is an $n \times 1$ vector of observation on dependent variable, $X$ is known design matrix of order $n \times p, \beta$ is a $p \times 1$ vector of unknown parameters and $\varepsilon$ is an $n \times 1$ vector of random errors with mean zero and variance $\sigma^{2} I_{n}$, where $I_{n}$ is an identity matrix of order $n$.

The OLS estimator (OLSE) of $\beta$ is given by

$$
\hat{\beta}=\left(X^{\prime} X\right)^{-1} X^{\prime} y,
$$

which depends on characteristics of the matrix $X^{\prime} X$. If $X^{\prime} X$ is ill-conditioned (near dependencies among various columns (regressors) of $X^{\prime} X$ exist) or $\operatorname{det}\left(X^{\prime} X\right) \approx 0$, then the OLS estimates are sensitive to a number of errors, such as non-significant or imprecise regression coefficients (Kmenta, 1980) with wrong sign and non-uniform eigenvalues spectrum. Moreover, the OLS method, can yield high variances of estimates, large standard errors, and wide confidence intervals. Quality and stability of the fitted model may be questionable due to erratic behaviour of the OLSE in case when regressors are collinear.

Researchers may tempt to eliminate regressor(s) causing the problem by consciously removing regressors from the model. However, this method may destroy the usefulness of the model by removing relevant regressor(s) from the model. To control variance and instability of the OLS estimates, one may regularize the coefficients, with some regularization methods such as ridge regression (RR), Liu regression, and Lasso regression methods etc., as alternative to OLS. Computationally, RR suppresses the effects of collinearity and reduces the apparent magnitude of the correlation among regressors in order to obtain more stable estimates of the coefficients than the OLS estimates and it also improves accuracy of prediction (see Hoerl and Kennard, 1970a; Montgomery and Peck, 1982; Myers, 1986; Rawlings et al., 1998; Seber and Lee, 2003; Tripp, 1983, etc.).

There are only a few software programs and $\mathrm{R}$ packages capable of estimating and/ or testing of ridge coefficients. The design goal of our lmridge (Imdad and Aslam, 2018b) is primarily to provide functionality of all possible ridge related computations. The output of our developed package (lmridge) is consistent with output of existing software/ $\mathrm{R}$ packages. The package, lmridge also provides the most complete suite of tools for ordinary RR, comparable to those listed in Table 1. For package development and R documentation, we followed Hadley (2015), Leisch (2008) and R Core Team (2015). The ridge package by Moritz and Cule (2017) and lm.ridge() from the MASS (Venables and Ripley, 2002) also provided guidance in coding.

All available software and $\mathrm{R}$ packages mentioned in Table 1 are compared with our lmridge package. For multicollinearity detection, NCSS statistical software (NCSS 11 Statistical Software, 2016) computes VIF/TOL, $R^{2}$, eigenvalue, eigenvector, incremental and cumulative percentage of eigenvalues and CN. For RR, ANOVA table, coefficient of variation, plot of residuals vs predicted, histogram and density trace of residuals are also available in NCSS. In SAS (Inc., 2011), collin option in the model statement is used to perform collinearity diagnostics while for remedy of multicollinearity, RR can be performed using a ridge option in proc reg statement. The outVIF option results in 


\begin{tabular}{|c|c|c|c|c|c|c|c|c|c|c|}
\hline & NCSS & SAS & Stata & StatGraphics & 1rmest & ltsbase & penalized & glmnet & ridge & lmridge \\
\hline \multicolumn{11}{|c|}{ Standardization of regressors } \\
\hline & $\checkmark$ & $\checkmark$ & $\checkmark$ & $\checkmark$ & & $\checkmark$ & $\checkmark$ & $\checkmark$ & $\checkmark$ & $\checkmark$ \\
\hline \multicolumn{11}{|c|}{ Estimation and testing of ridge coefficient } \\
\hline Estimation & $\checkmark$ & $\checkmark$ & $\checkmark$ & $\checkmark$ & $\checkmark$ & $\checkmark$ & $\checkmark$ & $\checkmark$ & $\checkmark$ & $\checkmark$ \\
\hline Testing & & & $\checkmark$ & & $\checkmark$ & & & & $\checkmark$ & $\checkmark$ \\
\hline SE of coef & $\checkmark$ & & $\checkmark$ & & $\checkmark$ & & & & $\checkmark$ & $\checkmark$ \\
\hline \multicolumn{11}{|c|}{ Ridge related statistics } \\
\hline$R^{2}$ & $\checkmark$ & & $\checkmark$ & $\checkmark$ & & & & & & $\checkmark$ \\
\hline $\operatorname{adj}-R^{2}$ & & & $\checkmark$ & $\checkmark$ & & & & & & $\checkmark$ \\
\hline m-scale \& ISRM & & & & & & & & & & $\checkmark$ \\
\hline Variance & & & & & & & & & & $\checkmark$ \\
\hline Bias $^{2}$ & & & & & & & & & & $\checkmark$ \\
\hline MSE & & & & & $\checkmark$ & $\checkmark$ & & & & $\checkmark$ \\
\hline F-test & & & $\checkmark$ & & & & & & & $\checkmark$ \\
\hline Shrinkage factor & & & & & & & & & & $\checkmark$ \\
\hline $\mathrm{CN}$ & & & & & & & & & & $\checkmark$ \\
\hline$\sigma^{2}$ & & & & & & & & & & $\checkmark$ \\
\hline $\mathrm{C}_{k}$ & & & & & & & & & & $\checkmark$ \\
\hline DF & & & & & & & & & & $\checkmark$ \\
\hline EDF & & & & & & & & & & $\checkmark$ \\
\hline Eft & & & & & & & & & & $\checkmark$ \\
\hline Hat matrix & & & & & & & & & & $\checkmark$ \\
\hline Var-Cov matrix & & & & & & & & & & $\checkmark$ \\
\hline VIF & $\checkmark$ & & & $\checkmark$ & & & & & $\checkmark$ & $\checkmark$ \\
\hline Residuals & $\checkmark$ & & $\checkmark$ & $\checkmark$ & & $\checkmark$ & $\checkmark$ & & & $\checkmark$ \\
\hline Ridge fitted & & & & & & $\checkmark$ & $\checkmark$ & & & $\checkmark$ \\
\hline Predict & $\checkmark$ & & $\checkmark$ & $\checkmark$ & & & $\checkmark$ & $\checkmark$ & $\checkmark$ & $\checkmark$ \\
\hline \multicolumn{11}{|c|}{ Ridge model selection } \\
\hline CV \& GCV & & & $\checkmark$ & & & & $\checkmark$ & $\checkmark$ & & $\checkmark$ \\
\hline AIC \& BIC & & & & & & & & & & $\checkmark$ \\
\hline PRESS & & & & & & & & & & $\checkmark$ \\
\hline \multicolumn{11}{|c|}{ Ridge related graphs } \\
\hline Ridge trace & $\checkmark$ & $\checkmark$ & & $\checkmark$ & & & & & $\checkmark$ & $\checkmark$ \\
\hline VIF trace & $\checkmark$ & $\checkmark$ & & $\checkmark$ & & & & & & $\checkmark$ \\
\hline Bias, var, MSE & & & & & & & & & & $\checkmark$ \\
\hline CV, GCV & & & & & & & & & & $\checkmark$ \\
\hline AIC \& BIC & & & & & & & & & & $\checkmark$ \\
\hline m-scale, ISRM & & & & & & & & & & $\checkmark$ \\
\hline DF, RSS, PRESS & & & & & & & & & & $\checkmark$ \\
\hline
\end{tabular}

Table 1: Comparison of ridge related software and $\mathrm{R}$ packages.

VIF values. For RR, Stata (StataCorp, 2014) has no built-in command, however ridgereg add-on is available that performs calculation on scalar k. The lrmest package (Dissanayake et al., 2016) computes estimators such as OLS, ordinary RR (ORR), Liu estimator (LE), LE type-1,2,3, Adjusted Liu Estimator (ALTE), and their type-1,2,3 etc. Moreover, lrmest provides scalar mean square error (MSE), prediction residual error sum of squares (PRESS) values of some of the estimators. The testing of ridge coefficient is performed only on scalar k, however, for vector of $k$, function rid() of lrmest package returns only MSE along with value of biasing parameter used. The function optimum() of lrmest package can be used to get the optimal scalar MSE and PRESS values (Arumairajan and Wijekoon, 2015). Statgraphics standardizes the dependent variable and computes some statistics for detection of collinearity such as $R^{2}$, adj- $R^{2}$, and VIF. Statgraphics also facilitates to perform RR and computes different RR related statistics such as VIF and ridge trace for different biasing parameter used, $R^{2}$, adj- $R^{2}$ and standard error of estimates etc. The ltsbase package (Kan-Kilinc and Alpu, 2013, 2015) computes ridge and Liu estimates based on the least trimmed squares (LTS) method. The MSE value from four regression models can be compared graphically if the argument plot=TRUE is passed to the ltsbase() function. There are three main functions (i) 1 tsbase() computes the minimum MSE values for six models: OLS, ridge, ridge based on LTS, LTS, Liu, and Liu based on LTS method for sequences of biasing parameters ranging from 0 to 1 . If print=TRUE, 1 tsbase () prints all the MSEs (along with minimum MSE) for ridge, Liu, and ridge \& Liu based on LTS method for the sequence of biasing parameters given by the user, (ii) the ltsbaseDefault() function returns the fitted values and residual of the six models (OLS, ridge, Liu, LTS, and ridge \& Liu based LTS methods) having minimum MSE, and (iii) the 1 tsbaseSummary() function returns the coefficients and the biasing parameter for the best MSE among the four regression models. The penalized package (Goeman et al., 2017) is designed for penalized estimation in generalized linear models. The supported models are linear regression, logistic 
regression, Poisson regression and the Cox proportional hazard models. The penalized package allows an L1 absolute value ("LASSO") penalty, and L2 quadratic ("ridge") penalty or a combination of the two. It is also possible to have a fused LASSO penalty with L1 absolute value penalty on the coefficients and their differences. The penalized package also includes facilities for likelihood, cross-validation and for optimization of the tuning parameter. The glmnet package (Friedman et al., 2010) has some efficient procedures for fitting the entire LASSO or elastic-net regularization path for linear regression, logistic and multinomial regression model, Poisson regression and Cox model. The glmnet can also be used to fit the RR model by setting alpha argument to zero. The ridge package fits linear and also logistic RR models, including functions for fitting linear and logistic RR models for genome-wide SNP data supplied as files names when the data are too big to read into R. The RR biasing parameter is chosen automatically using the method proposed by Cule and De Iorio (2012), however value of biasing parameter can also be specified for estimation and testing of ridge coefficients. The function, lm.ridge() from MASS only fits linear RR model and returns ridge biasing parameters given by Hoerl and Kennard (1970a) and Venables and Ripley (2002) and vector GCV criterion, given by Golub et al. (1979).

There are other software and R packages that can be used to perform RR analysis such as SPLUS (S-PLUS, 2008), Shazam (Shazam, 2011) and R packages such as RXshrink (Obenchain, 2014), rrBLUP (Endelman, 2011), RidgeFusion (Price, 2014), bigRR (Shen et al., 2013), lpridge (Seifert, 2013), genridge (Friendly, 2017) and CoxRidge (Perperoglou, 2015) etc.

This paper outlines the collinearity detection methods available in the existing literature and uses the mctest (Imdad and Aslam, 2018a) package through an illustrative example. To overcome the issues of the collinearity effect on regressors a thorough introduction to ridge regression, properties of the ridge estimator, different methods for selecting values of $k$, and testing of the ridge coefficients are presented. Finally, estimation of the ridge coefficients, methods of selecting a ridge biasing parameter, testing of the ridge coefficients, and different ridge related statistics are implemented in $\mathrm{R}$ within the lmridge.

\section{Collinearity detection}

Diagnosing collinearity is important to many researchers. It consists of two related but separate elements: (1) detecting the existence of collinear relationship among regressors and (2) assessing the extent to which this relationship has degraded the parameter estimates. There are many diagnostic measures used for detection of collinearity in the existing literature provided by various authors (Belsley et al., 1980; Curto and Pinto, 2011; Farrar and Glauber, 1967; Fox and Weisberg, 2011; Gunst and Mason, 1977; Imdadullah et al., 2016; Klein, 1962; Koutsoyiannis, 1977; Kovács et al., 2005; Marquardt, 1970; Theil, 1971). These diagnostics methods assist in determining whether and where some corrective action is necessary (Belsley et al., 1980). Widely used, and the most suggested diagnostics, are value of pair-wise correlations, variance inflation factor (VIF)/ tolerance (TOL) (Marquardt, 1970), eigenvalues and eigenvectors (Kendall, 1957), CN \& CI (Belsley et al., 1980; Chatterjee and Hadi, 2006; Maddala, 1988), Leamer's method (Greene, 2002), Klein's rule (Klein, 1962), the tests proposed by Farrar and Glauber (Farrar and Glauber, 1967), Red indicator (Kovács et al., 2005), corrected VIF (Curto and Pinto, 2011) and Theil's measures (Theil, 1971), (see also Imdadullah et al. (2016)). All of these diagnostic measures are implemented in the R package, mctest. Below, we use the Hald dataset (Hald, 1952), for testing collinearity among regressors. We then use the lmridge package to compute the ridge coefficients for different ridge related statistics and methods of selection of ridge biasing parameter is also performed. For optimal choice of ridge biasing parameter, graphical representations of the ridge coefficients, vif values, cross validation criteria (CV \& GCV), ridge DF, RSS, PRESS, ISRM and m-scale versus used ridge biasing parameter are considered. In addition graphical representation of model selection criteria (AIC \& BIC) of ridge regression versus ridge DF is also performed. The Hald data are about heat generated during setting of 13 cement mixtures of 4 basic ingredients and used by Hoerl et al. (1975). Each ingredient percentage appears to be rounded down to a full integer. The data set is already bundled in mctest and lmridge packages.

\section{Collinearity detection: Illustrative example}

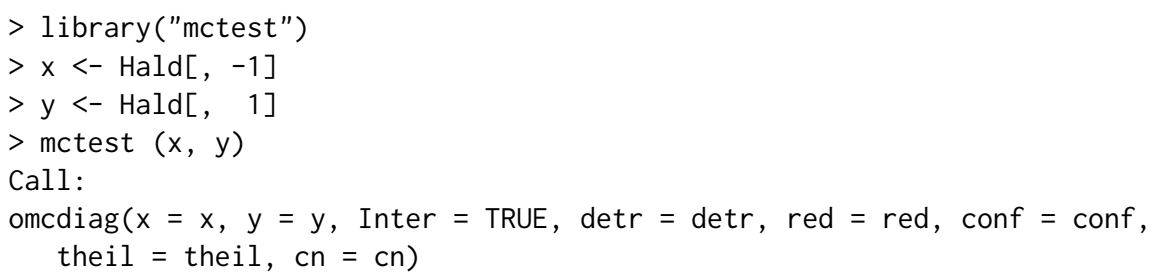


Overall Multicollinearity Diagnostics

$\begin{array}{lrr} & \text { MC Results detection } \\ \text { Determinant }\left|X^{\prime} \mathrm{X}\right|: & 0.0011 & 1 \\ \text { Farrar Chi-Square: } & 59.8700 & 1 \\ \text { Red Indicator: } & 0.5414 & 1 \\ \text { Sum of Lambda Inverse: } & 622.3006 & 1 \\ \text { Theil's Method: } & 0.9981 & 1 \\ \text { Condition Number: } & 249.5783 & 1 \\ & & \\ 1-->\text { COLLINEARITY is detected } & \\ 0-\rightarrow \text { COLLINEARITY is not detected by the test }\end{array}$

The results from all overall collinearity diagnostic measures indicate the existence of collinearity among regressor(s). These results do not tell which regressor(s) are reasons of collinearity. The individual collinearity diagnostic measures can be obtained through:

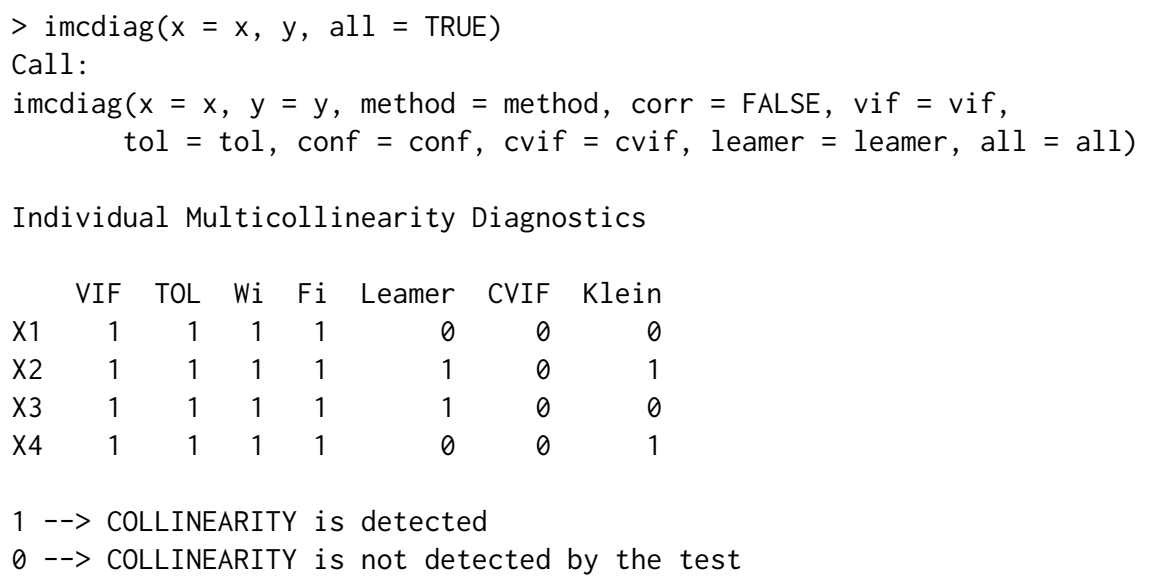

Results from the most of individual collinearity diagnostics suggest that all of the regressors are the reason for collinearity among regressors. The last line of imcdiag() function's output suggests that method argument should be used to check which regressors may be the reason of collinearity among different regressors. For further information about method argument, see the help file of imcdiag() function.

\section{Ridge regression analysis}

In the seminal work by Hoerl $(1959,1962,1964)$ and Hoerl and Kennard $(1970 b, a)$ have developed ridge analysis technique that purports the departure of the data from orthogonality. Hoerl (1962) introduced the RR, based on the James-Stein estimator by stating that existence of correlation among regressors can cause errors in estimating regression coefficients when applying the OLS method. The RR is similar to the OLS method however, it shrinks the coefficients towards zero by minimizing the MSE of the estimates, making the RR technique better than the OLSE with respect to MSE, when regressors are collinear with each other. A penalty (degree of bias) is imposed on the size of coefficients in the RR to reduce their variances. However, the expected values of these estimates are not equal to the true values and tend to under estimate the true parameter. Though the ridge estimators are biased but have lower MSE (more precision) than the OLSEs have, less sensitive to sampling fluctuations or model misspecification if number of regressors is more than the number of observations in a data set (i.e., $p>n$ ), and omitted variables specification bias (Theil, 1957). In summary, the RR procedure is intended to overcome the ill-conditioned situation, and is used to improve the estimation of regression coefficients when regressors are correlated and it also improves the accuracy of prediction (Seber and Lee, 2003). Obtaining the ridge model coefficients $\left(\hat{\beta}_{R}\right)$ is relatively straight forward, because the ridge coefficients are obtained by solving a slightly modified form of the OLS method. 
The design matrix $X$ in Eq. (1) can be standardized, scaled or centered. Usually, standardization of $X$ matrix is done as described by Belsley et al. (1980) and Draper and Smith (1998), that is, $X_{j}=$ $\frac{x_{i j}-\bar{x}_{j}}{\sqrt{\sum\left(x_{i j}-\bar{x}_{j}\right)^{2}}}$; where $j=1,2, \cdots, p$ such that $\bar{X}_{j}=0$ and $X_{j}^{\prime} X_{j}=1$, where $X_{j}$ is the $j$ th column of the matrix $X$. In this way, the new design matrix (say $\tilde{X}$ ) that contains the standardized $p$ columns and the matrix $\tilde{X}^{\prime} \tilde{X}$ will be correlation matrix of regressors. To avoid complexity of different notations and terms, the centered and scaled design matrix $\tilde{X}$ will be represented by $X$ and centered response variable as $y$.

The ridge model coefficients are estimated as,

$$
\hat{\beta}_{R_{k}}=\left(X^{\prime} X+k I_{p}\right)^{-1} X^{\prime} y,
$$

where $\hat{\beta}_{R_{k}}$ is the vector of standardized RR coefficients of order $p \times 1$ and $k I_{p}$ is a positive semi-definite matrix added to the $X^{\prime} X$ matrix. Note that for $k=0, \hat{\beta}_{R_{k}}=\hat{\beta}_{o l s}$.

The addition of constant term $k$ to diagonal element of $X^{\prime} X$ (in other words addition of $k I_{p}$ to $\left.X^{\prime} X\right)$ in Eq. (3) is known as penalty and $k$ is called the biasing or shrinkage parameter. Addition of this biasing parameter guarantees the invertibility of $X^{\prime} X$ matrix, such that there is always a unique solution $\hat{\beta}_{R_{k}}$ exists (Draper and Smith, 1998; Hoerl and Kennard, 1970a; McCallum, 1970) and the condition number $(\mathrm{CN})$ of $X^{\prime} X+k I\left(C N_{k}=\sqrt{\frac{\lambda_{1}+k I}{\lambda_{p}+k I}}\right)$ also becomes smaller as compared to that of $X^{\prime} X$, where $\lambda_{1}$ is the largest and $\lambda_{p}$ is the smallest eigenvalues of the correlation matrix $X^{\prime} X$. Therefore, the ridge estimator (RE) is an improvement over the OLSE for collinear data.

It is desirable to select the smallest value of $k$ for which stabilized regression coefficients occur and there always exists a particular value of $k$ for which the total MSE of the REs is less than the MSE of the OLSE, however, the optimum value of $k$ (which produces minimum MSE as compared to other values of $k \mathrm{~s}$ ) varies from one application to another and hence optimal value of $k$ is unknown. Any estimator that has a small amount of bias, less variance and substantially more precise than an unbiased estimator may be preferred since it will have larger probability of being close to the true parameter being estimated. Therefore, criterion of goodness of estimation considered in the RR is the minimum total MSE.

\section{Properties of the ridge estimator}

Let $X_{j}$ denotes the $j$ th column of $X(1,2, \cdots, p)$, where $X_{j}=\left(x_{1 j}, x_{2 j}, \cdots, x_{n j}\right)^{\prime}$. As already discussed, assume that the regressors are centered such that $\sum_{i=1}^{n} x_{i j}=0$ and $\sum_{i=1}^{n} x_{i j}^{2}=1$ and the response variable $y$ is centered.

The RR is the most popular among biased methods, because of its relationship to the OLS method and statistical properties of the RE are also well defined. Most of the RR properties have been discussed, proved and extended by many researchers such as Allen (1974); Hemmerle (1975); Hoerl and Kennard (1970b,a); Marquardt (1970); McDonald and Galarneau (1975); Newhouse and Oman (1971). Table 2 lists the RR properties.

Theoretically and practically, the RR is used to propose some new methods for the choice of the biasing parameter $k$ to investigate the properties of RE, since biasing parameter plays a key role while the optimal choice of $k$ is the main issue in this context. In the literature, there are many methods for estimating the biasing parameter $k$ (see Allen, 1974; Guilkey and Murphy, 1975; Hemmerle, 1975; Hoerl and Kennard, 1970b,a; McDonald and Galarneau, 1975; Obenchain, 1977; Hocking et al., 1976; Lawless and Wang, 1976; Vinod, 1976; Kasarda and Shih, 1977; Hemmerle and Brantle, 1978; Wichern and Churchill, 1978; Nordberg, 1982; Saleh and Kibria, 1993; Singh and Tracy, 1999; Wencheko, 2000; Kibria, 2003; Khalaf and Shukur, 2005; Alkhamisi et al., 2006; Alkhamisi and Shukur, 2007; Khalaf, 2013, among many more), however, there is no consensus about which method is preferable (Chatterjee and Hadi, 2006). Similarly, each of the estimation method of biasing parameter cannot guarantee to give a better $k$ or even cannot give a smaller MSE as compared to that for the OLS.

\section{Methods of selecting values of $k$}

The optimal value of $k$ is one which gives minimum MSE. There is one optimal $k$ for any problem, while a wide range of $k\left(0<k<k_{\text {opt }}\right)$ give smaller MSE as compared to that of the OLS. For collinear data, a small change in $k$ varies the RR coefficients rapidly. At some values of $k$, the ridge coefficients get stabilized and the rate of change slow down gradually to almost zero. Therefore, a disciplined way of selecting the shrinkage parameter is required that minimizes the MSE. The biasing parameter $k$ depends on the true regression coefficients $(\beta)$ and the variance of the residuals $\sigma^{2}$, unfortunately 


\begin{tabular}{|c|c|c|}
\hline sr.\# & Property & Formula \\
\hline 1) & Mean & $E\left(\hat{\beta}_{R}\right)=\left(X^{\prime} X+k I_{p}\right)^{-1} X^{\prime} X \beta$ \\
\hline 2) & Shorter regression coeffs. & $\hat{\beta}_{R}^{\prime} \hat{\beta}_{R} \leq \hat{\beta}^{\prime} \hat{\beta}$ \\
\hline 3) & Linear transformation & $\hat{\beta}_{R}=\bar{Z} \hat{\beta}$, where $Z=\left(X^{\prime} X+k I\right)^{-1} X^{\prime} X$ \\
\hline 4) & Variance & $\operatorname{Var}\left(\hat{\beta}_{R}\right)=\sigma^{2} \sum_{j=1}^{p} \frac{\lambda_{j}}{\left(\lambda_{j}+k\right)^{2}}$ \\
\hline 5) & Var-Cov matrix & $\begin{aligned} \operatorname{Cov}\left(\hat{\beta}_{R}\right) & =\operatorname{Cov}(Z \hat{\beta}) \\
& =\sigma^{2}\left(X^{\prime} X+k I\right)^{-1} X^{\prime} X\left(X^{\prime} X+k I\right)^{-1} \\
& =\sigma^{2}[V I F] \\
\operatorname{Bias}\left(\hat{\beta}_{R}\right) & =-k\left(X^{\prime} X+k I\right)^{-1} \beta\end{aligned}$ \\
\hline 6) & Bias & $=-k P \operatorname{diag}\left(\frac{1}{\lambda_{j}+k}\right) P^{\prime} \beta$ \\
\hline 7) & MSE & $M S E=\sigma^{2} \sum_{j=1}^{p} \frac{\lambda_{j}}{\left(\lambda_{j}+k\right)^{2}}+\sum_{j=1}^{p} \frac{k^{2} \alpha_{j}^{2}}{\left(\lambda_{j}+k\right)^{2}}$ \\
\hline $\begin{array}{l}\text { 8) } \\
\text { 9) }\end{array}$ & $\begin{array}{l}\text { Distance between } \hat{\beta}_{R} \text { and } \beta \\
\text { Inflated RSS }\end{array}$ & $\begin{array}{l}\hat{\beta}_{R} \text { and the true vector of } \beta \text { have minimum distance } \\
\phi_{0}=k^{2} \hat{\beta}_{R}^{\prime}\left(X^{\prime} X\right)^{-1} \hat{\beta}_{R}\end{array}$ \\
\hline 10) & $R_{R}^{2}$ & $R_{R}^{2}=\frac{\hat{\beta}_{R}^{\prime} X^{\prime} y-k \hat{\beta}_{R}^{\prime} \hat{\beta}_{R}}{y^{\prime} y}$ \\
\hline 11) & Sampling fluctuations & The $\hat{\beta}_{R}$ is less sensitive to the sampling fluctuation \\
\hline 12) & Accurate prediction & $\sigma_{f_{R}}^{2}=\sigma^{2}\left[1+x^{\prime} P \operatorname{diag}\left(\frac{\lambda_{j}}{(\lambda+k)^{2}}\right) P^{\prime} x\right]+\left(\operatorname{Bias}\left(\hat{\beta}_{R}\right)\right)^{2}$ \\
\hline 13) & Wide range of $k$ & $0<k<k_{\max }$, have smaller set of MSE than OLSE \\
\hline 14) & Optimal $k$ & An optimal $k$ always exists that gives minimum MSE \\
\hline 15) & DF Ridge & $\begin{array}{l}d f_{R_{k}}=E D F=\sum_{j=1}^{p} \frac{\lambda_{j}}{\lambda_{j}+k}=\operatorname{trace}\left[H_{R_{k}}\right] \\
\text { where } H_{R k}=X\left(X^{\prime} X+k I\right)^{-1} X^{\prime}\end{array}$ \\
\hline 16 & Effective no. of parameters & $E P=\operatorname{trace}\left[2 H_{R_{k}}-H_{R_{k}} H_{R_{k}}^{\prime}\right]$ \\
\hline 17 & Residual EDF & $R E D F=n-\operatorname{trace}\left[2 H_{R_{k}}-H_{R_{k}} H_{R_{k}}^{\prime}\right]=n-E P$ \\
\hline
\end{tabular}

Table 2: Properties of the ridge estimator.

these are unknown, but they can be estimated from the sample data.

We classified these estimation method as (i) Subjective or (ii) Objective

\section{Subjective methods}

In all these methods, the selection of $k$ is subjective or of judgmental nature and provides graphical evidence of the effect of collinearity on the regression coefficient estimates and also accounts for variation by the RE as compared to the OLSE. In these methods, the reasonable choice of $k$ is done using the ridge trace, df trace, VIF trace and plotting of bias, variance, and MSE. The ridge trace is a graphical representation of regression coefficients $\hat{\beta}_{R}$, as a function of $k$ over the interval $[0,1]$. The $\mathrm{df}$ trace and VIF trace are like the ridge trace plot in which EDF and VIF values are plotted against $k$. Similarly, plotting of bias, variance, and MSE from the RE may also be helpful in selecting an appropriate value of $k$. All these graphs can be used for selection of optimal (but judgmental) value of $k$ from horizontal axis to assess the effect of collinearity on each of the coefficients. The effect of collinearity is depressed when value of $k$ increases and all the values of the ridge coefficients, EDF and VIF values decrease and/ or may stabilize after certain value of $k$. These graphical representations do not provide a unique solution, rather they render a vaguely defined class of acceptable solutions. However, these traces are still useful graphical representations to check for some optimal $k$.

\section{Objective methods}

Suppose, we have set of observations $\left(x_{1}, y_{1}\right),\left(x_{2}, y_{2}\right), \cdots,\left(x_{n}, y_{n}\right)$ and the RR model as given in Eq. (3). Objective methods, to some extent, are similar to judgmental methods for selection of biasing parameter $k$, but they require some calculations to obtain these biasing parameters. Table 3 lists widely used methods to estimate the biasing parameter $k$ already available in the existing literature. Table 3 
also lists other statistics that can be used for the selection of the biasing parameter $k$. There are other

\begin{tabular}{|c|c|c|}
\hline method & formula & reference \\
\hline$C_{k}$ & $\begin{aligned} C_{k} & =\frac{S S R_{k}}{s^{2}}-n+2+2 \operatorname{trace}\left(H_{R_{k}}\right) \\
& =\frac{S S R_{k}}{s^{2}}+2\left(1+\operatorname{trace}\left(H_{R_{k}}\right)\right)-n\end{aligned}$ & $\begin{array}{l}\text { Kennard (1971); Mallows } \\
\text { (1973) }\end{array}$ \\
\hline$P_{R E S S_{k}}$ & $\begin{aligned} \text { PRESS }_{k} & =\sum_{i=1}^{n}\left(y_{i}-\hat{y}_{(i,-i)_{k}}\right)^{2} \\
& =\sum_{i=1}^{n} e_{(i,-i)_{k}}^{2}\end{aligned}$ & Allen $(1971,1974)$ \\
\hline CV & $C V_{k}=n^{-1} \sum_{i=1}^{n}\left(y_{i}-X_{j} \hat{\beta}_{j_{R_{k}}}\right)^{2}$ & $\begin{array}{l}\text { Delaney and Chatterjee } \\
\text { (1986) }\end{array}$ \\
\hline GCV & $G C V_{k}=\frac{S R R_{k}}{n-\left(1+\operatorname{trace}\left(H_{R_{k}}\right)\right)^{2}}$ & Golub et al. (1979) \\
\hline ISRM & $\operatorname{ISRM}_{k}=\sum_{j=1}^{p}\left(\frac{p\left(\frac{\lambda_{j}}{\lambda_{j}+k}\right)^{2}}{\sum_{j=1}^{p} \frac{\lambda_{j}}{\left(\lambda_{j}+k\right)^{2}} \lambda_{j}}-1\right)^{2}$ & Vinod (1976) \\
\hline m-scale & $m=p-\sum_{j=1}^{p} \frac{\lambda_{j}}{\lambda_{j}+k}$ & Vinod (1976) \\
\hline Information criteria & $\begin{array}{l}A I C=n \cdot \log (R S S / n)+2 \cdot d f_{R k} \\
B I C=n \cdot \log (R S S)+2 \cdot d f_{R k}\end{array}$ & $\begin{array}{l}\text { Akaike (1973); Schwarz } \\
\text { (1978) }\end{array}$ \\
\hline Effectiveness index (Eft) & $E F=\frac{\sigma^{2} \operatorname{trace}\left(X^{\prime} X\right)^{-1}-\sigma^{2} \operatorname{trace}(V I F)}{\left(\operatorname{Bias}\left(\hat{\beta}_{R}\right)\right)^{2}}$ & Lee (1979) \\
\hline
\end{tabular}

Table 3: Objective methods for selection of biasing parameter $k$.

methods to estimate biasing parameter $k$. Table 4 lists various methods for the selection of biasing parameter $k$, proposed by different researchers.

\section{Testing of the ridge coefficients}

Investigating of the individual coefficients in a linear but biased regression models such as ridge based, exact and non-exact $t$ type and $F$ test can be used. Exact $t$-statistics derived by Obenchain (1977) based on the RR for matrix $G$ whose columns are the normalized eigenvectors of $X^{\prime} X$, is,

$$
t^{*}=\frac{\hat{\beta}_{R_{j}}-\beta_{j}}{\sqrt{\operatorname{var} r\left(\hat{\beta}_{R_{j}}-\beta_{j}\right)}},
$$

where $j=1,2, \cdots, p, v \hat{a} r\left(\hat{\beta}_{R_{j}}-\beta_{j}\right)$ is an unbiased estimator of the variance of the numerator in Eq. (4), and

$$
\beta_{j}=g_{i}^{\prime} \Delta G^{\prime}\left[I-\left(X^{\prime} X\right)^{-1} e_{i}^{\prime}\left(e_{i}\left(X^{\prime} X\right)^{-1} e_{i}^{\prime}\right)^{-1}\right] \hat{\beta}(0),
$$

where $g_{i}^{\prime}$ is the $i$ th row of $G, \Delta$ is the $(p \times p)$ diagonal matrix with $i$ th diagonal element given by $\delta_{i}=\frac{\lambda_{i}}{\lambda_{i}+k}$ and $e_{i}$ is the $i$ th row of the identity matrix.

It has been established that $\beta_{R} \sim N\left(Z X \beta, \phi=Z \Omega Z^{\prime}\right)$, where $Z=\left(X^{\prime} X+k I_{p}\right)^{-1} X^{\prime}$. Therefore, for $j$ th ridge coefficient $\beta_{R} \sim N\left(Z_{j} X \beta, \phi_{j j}=Z_{j} \Omega Z_{j}^{\prime}\right)$ (see Aslam, 2014; Halawa and El-Bassiouni, 2000). Halawa and El-Bassiouni (2000) presented to tackle the problem of testing $H_{0}: \beta_{j}=0$ by considering a non-exact $t$ type test of the form,

$$
t_{R_{j}}=\frac{\hat{\beta}_{R_{j}}}{\sqrt{S^{2}\left(\hat{\beta}_{R_{j}}\right)}},
$$

where $\hat{\beta}_{R_{j}}$ is the $j$ th element of RE and $S^{2}\left(\hat{\beta}_{R_{j}}\right)$ is an estimate of the variance of $\hat{\beta}_{R_{j}}$ given by the $i$ th diagonal element of the matrix $\sigma^{2}\left(X^{\prime} X+k I_{p}\right)^{-1} X^{\prime} X\left(X^{\prime} X+k I_{p}\right)^{-1}$. 


\begin{tabular}{|c|c|c|}
\hline Sr. \# & Formula & Reference \\
\hline 1) & $K_{H K B}=\frac{p \hat{\sigma}^{2}}{\hat{\beta}^{\prime} \hat{\beta}}$ & Hoerl and Kennard (1970a) \\
\hline 2) & $K_{T H}=\frac{(p-2) \hat{\sigma} 2}{\hat{\beta}^{\prime} \hat{\beta}}$ & Thisted (1976) \\
\hline 3) & $K_{L W}=\frac{p \hat{\sigma}^{2}}{\sum_{j=1}^{p} \lambda_{j} \hat{\alpha}_{j}^{2}}$ & Lawless and Wang (1976) \\
\hline 4) & $K_{D S}=\frac{\hat{\sigma}^{2}}{\hat{\beta}^{\prime} \hat{\beta}}$ & Dwividi and Shrivastava (1978) \\
\hline 5) & $K_{L W}=\frac{(p-2) \hat{\sigma}^{2} \times n}{\hat{\beta}^{\prime} X^{\prime} X \hat{\beta}}$ & Venables and Ripley (2002) \\
\hline 6) & $K_{A M}=\frac{1}{p} \sum_{j=1}^{p}{\frac{\hat{\sigma}^{2}}{\hat{\alpha}}}_{j}$ & Kibria (2003) \\
\hline 7) & $\hat{K}_{G M}=\frac{\hat{\sigma}^{2}}{\left(\frac{p}{n} \hat{\hat{N}}^{2}\right)^{\frac{1}{p}}}$ & Kibria (2003) \\
\hline 8) & $\hat{K}_{M E D}=\operatorname{Median}\left\{\frac{\hat{\sigma}^{2}}{\hat{\alpha}_{j}^{2}}\right\}$ & Kibria (2003) \\
\hline 9) & $K_{K M 2}=\max \mid \frac{1}{\sqrt{\frac{\hat{\sigma}^{2}}{\hat{\tilde{\alpha}}_{i}^{2}}}}$ & Muniz and Kibria (2009) \\
\hline 10) & $K_{K M 3}=\max \left(\sqrt{\frac{\sigma_{j}^{2}}{\hat{\alpha}_{j}^{2}}}\right)$ & Muniz and Kibria (2009) \\
\hline 11) & $K_{K M 4}=\mid \prod_{j=1}^{p} \frac{1}{\sqrt{\sqrt{\frac{\hat{\sigma}_{j}^{2}}{\hat{\alpha}_{i}^{2}}}}}$ & Muniz and Kibria (2009) \\
\hline 12) & $K_{K M 5}=\left(\prod_{j=1}^{p} \sqrt{\frac{\hat{\sigma}_{j}^{2}}{\hat{\alpha}_{j}^{2}}}\right)^{r}$ & Muniz and Kibria (2009) \\
\hline 13) & $K_{K M 6}=$ Median $\left(\frac{1}{\sqrt{\frac{\hat{\sigma}_{j}^{2}}{\hat{\alpha}_{j}^{2}}}}\right)$ & Muniz and Kibria (2009) \\
\hline 14) & $K_{K M 8}=\max \left(\frac{1}{\sqrt{\frac{\lambda_{\max \hat{\sigma}^{2}}}{(n-p) \hat{\sigma}^{2}+\lambda_{\max \hat{\alpha}_{j}^{2}}}}}\right)$ & Muniz et al. (2012) \\
\hline 15) & $K_{K M 9}=\max \left(\sqrt{\frac{\lambda_{\max } \hat{\sigma}^{2}}{(n-p) \hat{\sigma}^{2}+\lambda_{\max } \hat{\alpha}_{j}^{2}}}\right)$ & Muniz et al. (2012) \\
\hline 16) & $K_{K M 10}=\left(\prod_{j=1}^{p} \frac{1}{\sqrt{\frac{\lambda \max \hat{\sigma}^{2}}{(n-p) \tilde{\sigma}^{2}+\lambda_{\max \hat{\alpha}_{j}^{2}}}}}\right)$ & Muniz et al. (2012) \\
\hline 17) & $K_{K M 11}=\left(\prod_{j=1}^{p} \sqrt{\frac{\lambda_{\max } \hat{\sigma}^{2}}{(n-p) \hat{\sigma}^{2}+\lambda_{\max } \hat{\alpha}_{j}^{2}}}\right)^{\bar{p}}$ & Muniz et al. (2012) \\
\hline 18) & $K_{K M 12}=$ Median $\left(\frac{1}{\sqrt{\frac{\lambda \max \hat{\sigma}^{2}}{(n-p) \hat{\sigma}^{2}+\lambda_{\max \hat{\alpha}_{j}^{2}}}}}\right)$ & Muniz et al. (2012) \\
\hline 19) & $K_{K D}=\max \left(0, \frac{p \hat{\sigma}^{2}}{\hat{\alpha}^{\prime} \hat{\alpha}}-\frac{1}{n\left(V I F_{j}\right)_{\max }}\right)$ & Dorugade and Kashid (2010) \\
\hline 20) & $\begin{aligned} K_{4(A D)} & =\text { Harmonic Mean }\left[K_{i}(A D)\right] \\
& =\frac{2 p}{\lambda_{\max }} \sum_{j=1}^{p} \frac{\hat{\sigma}^{2}}{\hat{\alpha}_{j}^{2}}\end{aligned}$ & Dorugade (2014) \\
\hline
\end{tabular}

Table 4: Different available methods to estimate $k$. 
The statistic $t_{R_{j}}$ is assumed to follow a Student's $t$ distribution with $(n-p)$ d.f. (Halawa and El-Bassiouni, 2000). Hastie and Tibshirani (1990); Cule and De Iorio (2012) suggested to use [ $n-$ $\left.\operatorname{trace}\left(H_{R k}\right)\right]$ d.f. For large sample size, the asymptotic distribution of this statistic is normal (Halawa and El-Bassiouni, 2000). Thus, $H_{0}$ is rejected when $|T|>Z_{1-\frac{\alpha}{2}}$.

Similarly, for testing the hypothesis $H_{0}: \beta \neq \beta_{0}$, where $\beta_{0}$ is vector of fixed values. The $F$ statistic for significance testing of the ORR estimator $\beta_{R}$ with $E\left(\hat{\beta}_{R}\right)=Z X \beta$ and estimate of $\operatorname{Cov}\left(\beta_{R}\right)$ distributed as $F\left(D F_{\text {ridge }}, R E D F\right)$ is

$$
F=\frac{1}{p}\left(\hat{\beta}_{R}-Z X \beta\right)^{\prime}\left(\operatorname{Cov}\left(\hat{\beta}_{R}\right)\right)^{-1}\left(\hat{\beta}_{R}-Z X \beta\right)
$$

\section{The $\mathbf{R}$ package lmridge}

Our R package lmridge contains functions related to fitting of the RR model and provides a simple way of obtaining the estimates of RR coefficients, testing of the ridge coefficients, and computation of different ridge related statistics, which prove helpful for selection of optimal biasing parameter $k$. The package computes different ridge related measures available for the selection of biasing parameter $k$, and also computes value of different biasing parameters proposed by some researchers in the literature.

The lmridge objects contain a set of standard methods such as print (), summary(), plot () and predict(). Therefore, inferences can be made easily using summary() method for assessing the estimates of regression coefficients, their standard errors, $t$ values and their respective $p$ values. The default function $1 \mathrm{mr}$ idge which calls $1 \mathrm{mr}$ idgeEst () to perform required computations and estimation for given values of non-stochastic biasing parameter $k$. The syntax of default function is,

lmridge (formula, data, scaling = ("sc", "scaled", "centered") , K, ..)

The four arguments of lmridge() are described in Table 5:

\begin{tabular}{ll}
\hline Argument & Description \\
\hline formula & Symbolic representation for RR model of the form, response $\sim$ predictors. \\
data & Contains the variables that have to be used in RR model. \\
$\mathrm{K}$ & The biasing parameter, may be a scalar or vector. If a $K$ value is not pro- \\
& vided, $K=0$ will be used as the default value, i.e., the OLS results will be \\
& produced. \\
scaling & The methods for scaling the predictors. The sc option uses the default \\
& scaling of the predictors in correlation form as described in (Belsley, 1991; \\
& $\begin{array}{l}\text { Draper and Smith, 1998); the scaled option standardizes the predictors } \\
\text { having zero mean and unit variance; and the centered option centers the } \\
\text { predictors. }\end{array}$ \\
\hline
\end{tabular}

Table 5: Description of lmridge() function arguments.

The lmridge() function returns an object of class "lmridge". The function summary (), kest (), and kstats 1() etc., are used to compute and print a summary of the RR results, list of biasing parameter given in Table 4 , and ridge related statistics such as estimated squared bias, $R^{2}$ and variance etc., after addition of $k$ to diagonal of $X^{\prime} X$ matrix. An object of class "lmridge" is a list, the components of which are described in Table 6:

Table 7 lists the functions and methods available in lmridge package:

\section{The lmridge package implementation in $\mathbf{R}$}

The use of lmridge is explained through examples by using the Hald dataset.

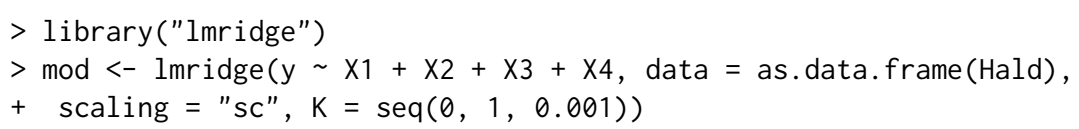

The output of linear RR from lmridge() function is assigned to an object mod. The first argument of the function is formula, which is used to specify the required linear RR model for the data provided as second argument. The print method for mod, an object of class "lmridge", will display the de-scaled coefficients. The output (de-scaled coefficients) from the above command is only for a few selected biasing parameter values. 


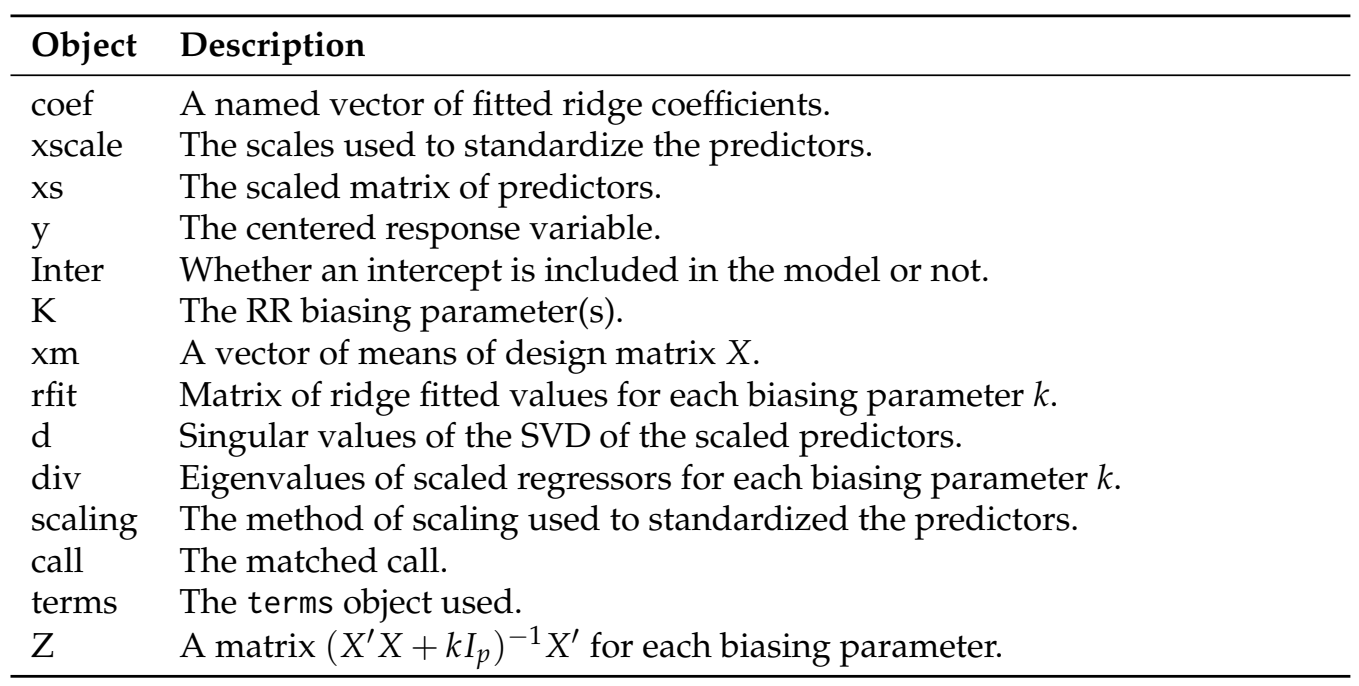

Table 6: Objects from "lmridge" class.

Call:

lmridge.default (formula $=\mathrm{y} \sim$., data $=$ as.data.frame $(\mathrm{Hald})$, $\mathrm{K}=\operatorname{seq}(0,1,0.001))$

$\begin{array}{lllll}\text { Intercept } & \mathrm{X} 1 & \mathrm{X} 2 & \mathrm{X} 3 & \mathrm{X} 4\end{array}$

$\mathrm{K}=0.01 \quad 82.67556 \quad 1.31521 \quad 0.30612 \quad-0.12902-0.34294$

$\mathrm{K}=0.05 \quad 85.83062 \quad 1.19172 \quad 0.28850-0.21796-0.35423$

$\mathrm{K}=0.5 \quad 89.19604 \quad 0.78822 \quad 0.27096-0.36391 \quad-0.28064$

$\mathrm{K}=0.9 \quad 90.22732 \quad 0.65351 \quad 0.24208-0.34769-0.24152$

$\mathrm{K}=1 \quad 90.42083 \quad 0.62855 \quad 0.23540 \quad-0.34119-0.23358$

To get the ridge scaled coefficients mod $\$ c o e f$ can be used,

$\begin{array}{lrrrrr}>\text { mod } \$ \text { coef } \\ \text { K=0.01 } & \mathrm{K}=0.05 & \mathrm{~K}=0.5 & \mathrm{~K}=0.9 & \mathrm{~K}=1 \\ \mathrm{X} 1 & 26.800306 & 24.28399 & 16.061814 & 13.316802 & 12.808065 \\ \mathrm{X} 2 & 16.500987 & 15.55166 & 14.606166 & 13.049400 & 12.689060 \\ \mathrm{X} 3 & -2.862655 & -4.83610 & -8.074509 & -7.714626 & -7.570415 \\ \mathrm{X} 4 & -19.884534 & -20.53939 & -16.272482 & -14.004088 & -13.543744\end{array}$

Objects of class "lmridge" contain components such as rfit, $\mathrm{K}$ and coef etc. For fitted ridge model, the generic method summary() is used to investigate the ridge coefficients. The parameter estimates of ridge model are summarized using a matrix of 5 columns namely estimates, estimates (Sc), StdErr (Sc), $t$ values $(S c)$ and $P(>|t|)$ for ridge coefficients. The following results are shown only for $K=0.012$ which produces minimum MSE as compared to others values specified in the argument.

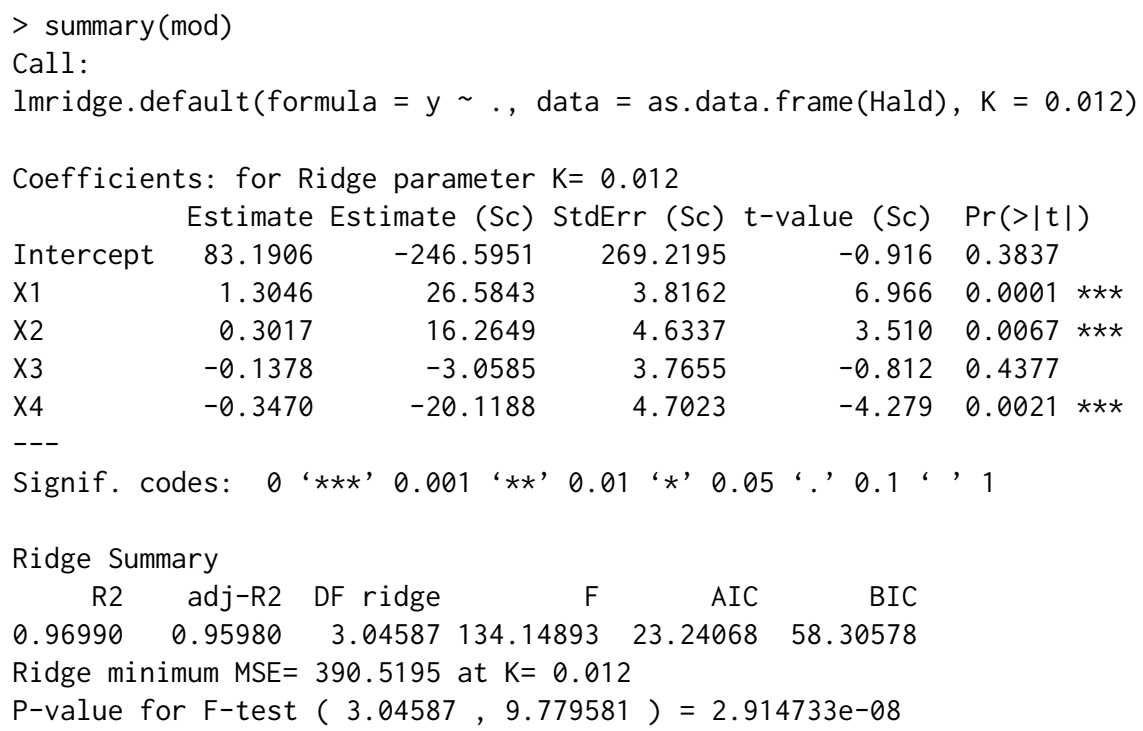




\begin{tabular}{|c|c|}
\hline Functions & Description \\
\hline \multicolumn{2}{|c|}{ Ridge coefficient estimation and testing } \\
\hline $\begin{array}{l}\text { lmridgeEst() } \\
\operatorname{coef}()\end{array}$ & $\begin{array}{l}\text { The main model fitting function for implementation of RR models in R. } \\
\text { Display de-scaled ridge coefficients. }\end{array}$ \\
\hline lmridge() & $\begin{array}{l}\text { Generic function and default method that calls lmridgeEst }() \text { and returns } \\
\text { an object of S3 class "lmridge" with different set of methods to standard } \\
\text { generics. It has a print method for display of ridge de-scaled coefficients. }\end{array}$ \\
\hline summary () & $\begin{array}{l}\text { Standard RR output (coefficient estimates, scaled coefficients estimates, } \\
\text { standard errors, } t \text { values and } p \text { values); returns an object of class "sum- } \\
\text { maryridge" containing the relative summary statistics and has a print } \\
\text { method. }\end{array}$ \\
\hline \multicolumn{2}{|c|}{ Residuals, fitted values and prediction } \\
\hline predict () & $\begin{array}{l}\text { Produces predicted value(s) by evaluating } \operatorname{lmridgeEst}() \text { in the frame } \\
\text { newdata. }\end{array}$ \\
\hline fitted() & Displays ridge fitted values for observed data. \\
\hline residuals() & Displays ridge residuals values. \\
\hline press() & $\begin{array}{l}\text { Generic function that computes prediction residual error sum of squares } \\
\text { (PRESS) for ridge coefficients. }\end{array}$ \\
\hline \multicolumn{2}{|c|}{ Methods to estimate $k$} \\
\hline $\operatorname{kest}()$ & $\begin{array}{l}\text { Displays various } k \text { (biasing parameter) values from different authors } \\
\text { available in literature and have a print method. }\end{array}$ \\
\hline $\begin{array}{l}\text { Ridge statistics } \\
\operatorname{vcov}()\end{array}$ & $\begin{array}{l}\text { Displays associated Var-Cov matrix with matching ridge parameter } k \\
\text { values }\end{array}$ \\
\hline hatr () & Generic function that displays hat matrix from RR. \\
\hline infocr () & Generic function that compute information criteria AIC and BIC. \\
\hline $\operatorname{vif}()$ & Generic function that computes VIF values. \\
\hline rstats1() & $\begin{array}{l}\text { Generic function that displays different statistics of RR such as MSE, } \\
\text { squared bias and } R^{2} \text { etc., and have print method. }\end{array}$ \\
\hline rstats2() & $\begin{array}{l}\text { Generic function that displays different statistics of RR such as } \mathrm{df}, \mathrm{m} \text {-scale } \\
\text { and LSRM etc., and have print method. }\end{array}$ \\
\hline \multicolumn{2}{|l|}{ Ridge plots } \\
\hline $\operatorname{plot}()$ & Ridge and VIF trace plot against biasing parameter $k$ \\
\hline bias.plot() & Bias-Variance tradeoff plot. Plot of ridge MSE, bias and variance against $k$ \\
\hline cv.plot() & Cross validation plots of CV and GCV against biasing parameter $k$. \\
\hline info.plot() & Plot of AIC and BIC against $k$. \\
\hline isrm.plot() & Plots ISRM and m-scale measure. \\
\hline rplots.plot() & Miscellaneous ridge related plots such as df-trace, RSS and PRESS plots. \\
\hline
\end{tabular}

Table 7: Functions and methods in lmridge package.

The summary() function also displays ridge related $R^{2}$, adjusted- $R^{2}, \mathrm{df}, F$ statistics, AIC, BIC and minimum MSE at certain $k$ given in $\operatorname{lmridge}()$.

The kest () function, which works with ridge fitted model, computes different biasing parameters developed by researchers, see Table 4 . The list of different $k$ values ( 22 in numbers) may help in deciding the amount of bias needs to be introduced in RR.

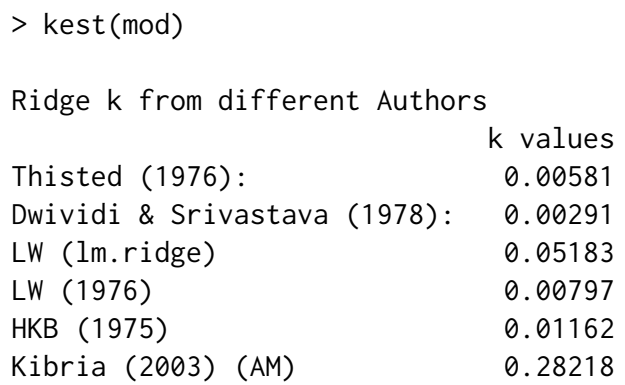




$\begin{array}{lr}\text { Minimum GCV at } & 0.01320 \\ \text { Minimum CV at } & 0.01320 \\ \text { Kibria 2003 (GM): } & 0.07733 \\ \text { Kibria 2003 (MED): } & 0.01718 \\ \text { Muniz et al. 2009 (KM2): } & 14.84574 \\ \text { Muniz et al. 2009 (KM3): } & 5.32606 \\ \text { Muniz et al. 2009 (KM4): } & 3.59606 \\ \text { Muniz et al. 2009 (KM5): } & 0.27808 \\ \text { Muniz et al. 2009 (KM6): } & 7.80532 \\ \text { Mansson et al. 2012 (KMN8): } & 14.98071 \\ \text { Mansson et al. 2012 (KMN9): } & 0.49624 \\ \text { Mansson et al. 2012 (KMN10): } & 6.63342 \\ \text { Mansson et al. 2012 (KMN11): } & 0.15075 \\ \text { Mansson et al. 2012 (KMN12): } & 8.06268 \\ \text { Dorugade et al. 2010: } & 0.00000 \\ \text { Dorugade et al. 2014: } & 0.00000\end{array}$

The rstats1() and rstats2() functions can be used to compute different statistics for a given ridge biasing parameter specified in a call to $1 \mathrm{mridge}$. The ridge statistics are MSE, squared bias, $F$ statistics, ridge variance, degrees of freedom by Hastie and Tibshirani (1990), condition numbers, PRESS, $R^{2}$, and ISRM etc. Following are the results using rstats1() and rstats2() functions, for some $(K=0,0.012,0.1,0.2)$.

$>\operatorname{rstats} 1$ (mod)

Ridge Regression Statistics 1:

$\begin{array}{lrrrrrrrr} & \text { Variance } & \text { Bias^2 }^{\wedge} & \text { MSE } & \text { rsigma2 } & F & \text { R2 } & \text { adj-R2 } & \text { CN } \\ \text { K=0 } & 3309.5049 & 0.0000 & 3309.5049 & 5.3182 & 125.4142 & 0.9824 & 0.9765 & 1376.8806 \\ \mathrm{~K}=0.012 & 72.3245 & 318.1951 & 390.5195 & 4.9719 & 134.1489 & 0.9699 & 0.9598 & 164.9843 \\ \mathrm{~K}=0.1 & 19.8579 & 428.4112 & 448.2692 & 5.8409 & 114.1900 & 0.8914 & 0.8552 & 22.9838 \\ \mathrm{~K}=0.2 & 16.5720 & 476.8887 & 493.4606 & 7.6547 & 87.1322 & 0.8170 & 0.7560 & 12.0804\end{array}$

$>\operatorname{rstats} 2(\mathrm{mod})$

Ridge Regression Statistics 2:

\begin{tabular}{lrrrrrrrr} 
& \multicolumn{1}{c}{ CK DF ridge } & EP & REDF & EF & ISRM & $\mathrm{m}$ scale & PRESS \\
$\mathrm{K}=0$ & 6.0000 & 4.0000 & 4.0000 & 9.0000 & 0.0000 & 3.9872 & 0.0000 & 110.3470 \\
$\mathrm{~K}=0.012$ & 4.8713 & 3.0459 & 3.2204 & 9.7796 & 10.1578 & 3.6181 & 0.9541 & 92.8977 \\
$\mathrm{~K}=0.1$ & 4.2246 & 2.5646 & 2.9046 & 10.0954 & 7.6829 & 2.8471 & 1.4354 & 121.2892 \\
$\mathrm{~K}=0.2$ & 3.8630 & 2.2960 & 2.7290 & 10.2710 & 6.9156 & 2.5742 & 1.7040 & 162.2832
\end{tabular}

The residuals, fitted values from the RR and predicted values of the response variable $y$ can be computed using functions residual(), fitted() and predict(), respectively. To obtain the Var-Cov matrix, VIF and Hat matrix, the function $\operatorname{vcov}(), \operatorname{vif}()$ and hatr () can be used. The df are computed by following Hastie and Tibshirani (1990). The results for VIF, Var-Cov and diagonal elements of the hat matrix from $\operatorname{vif}(), \operatorname{vcov}()$ and hatr() functions are given below for $K=0.012$.

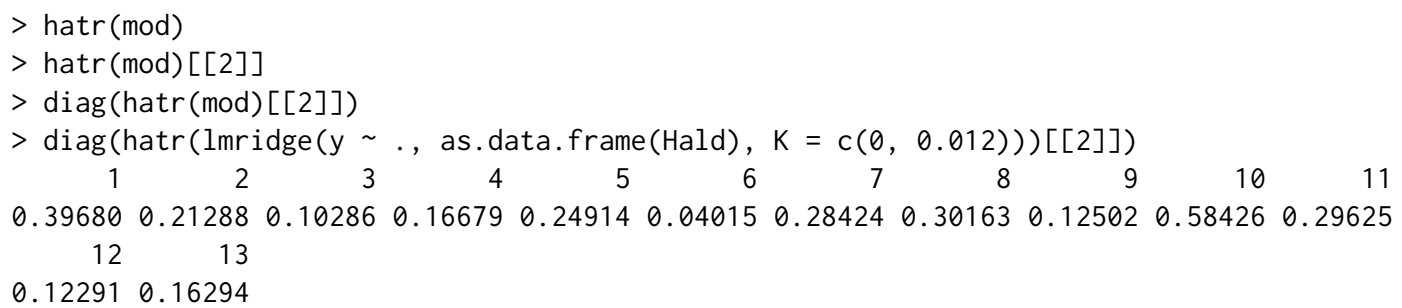

$>\operatorname{vif}(\bmod )$

$\begin{array}{lrrrr} & & & & X \\ \text { k=0 } & 38.49621 & 254.42317 & 46.86839 & 282.51286 \\ k=0.012 & 2.92917 & 4.31848 & 2.85177 & 4.44723 \\ k=0.1 & 1.28390 & 0.51576 & 1.20410 & 0.39603 \\ k=0.2 & 0.78682 & 0.34530 & 0.75196 & 0.28085\end{array}$

$\mathrm{R}>\mathrm{v} \operatorname{cov}(\mathrm{mod})$

$\${ }^{`} \mathrm{~K}=0.012^{\prime}$ 


$\begin{array}{rrrrr} & & & & \\ \text { X1 } & \text { X2 } & \text { X3 } & \text { X4 } \\ \text { X1 } & 14.563539 & 1.668783 & 11.577483 & 4.130232 \\ \text { X2 } & 1.668783 & 21.471027 & 3.066958 & 19.075274 \\ \text { X3 } & 11.577483 & 3.066958 & 14.178720 & 4.598000 \\ \text { X4 } & 4.130232 & 19.075274 & 4.598000 & 22.111196\end{array}$

Following are possible uses of some functions to compute different ridge related statistics. For detail description of these functions / commands, see the lmridge package documentation.

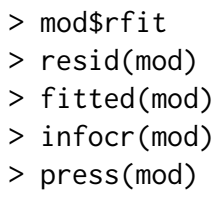

For given values of $X$, such as for first five rows of $X$ matrix, the predicted values for some $K=$ $0,0.012,0.1$, and 0.2 will be computed by predict():

$\begin{array}{rrrrrr}> & \text { predict(mod, newdata }= & \text { as. data. frame }(\mathrm{Hald}[1: & 5,-1])) \\ & \mathrm{K}=0 & \mathrm{~K}=0.012 & \mathrm{~K}=0.1 & \mathrm{~K}=0.2 \\ 1 & 78.49535 & 78.52225 & 79.75110 & 80.73843 \\ 2 & 72.78893 & 73.13500 & 74.32678 & 75.38191 \\ 3 & 105.97107 & 106.39639 & 106.04958 & 105.62451 \\ 4 & 89.32720 & 89.48443 & 89.52343 & 89.65432 \\ 5 & 95.64939 & 95.73595 & 96.56710 & 96.99781\end{array}$

The model selection criteria's of AIC and BIC can be computed using infocr () function for each value of $K$ used in argument of ridge(). For some $K=0,0.012,0.1$, and 0.2 , the AIC and BIC values are:

$\begin{array}{lrr}>\text { infocr(mod) } & \\ \text { K=0 } & 24.94429 & 60.54843 \\ \mathrm{~K}=0.012 & 23.24068 & 58.30578 \\ \mathrm{~K}=0.1 & 24.78545 & 59.57865 \\ \mathrm{~K}=0.2 & 27.98813 & 62.62961\end{array}$

The effect of multicollinearity on the coefficient estimates can be identified by using different graphical displays such as ridge, VIF and df traces, plotting of RSS against df, PRESS vs $k$, and the plotting of bias, variance, and MSE against $K$ etc. Therefore, for selection of optimal $k$ using subjective (judgmental) methods, different plot functions are also available in lmridge package. For example, the ridge (Figure 1) or vif trace (Figure 2) can be plotted using plot () function. The argument to plot functions are abline $=$ TRUE, and type $=c(" r i d g e ", " v i f ")$. By default, ridge trace will be plotted having horizontal line parallel to horizontal axis at $y=0$ and vertical line on $x$-axis at $k$ having minimum GCV.

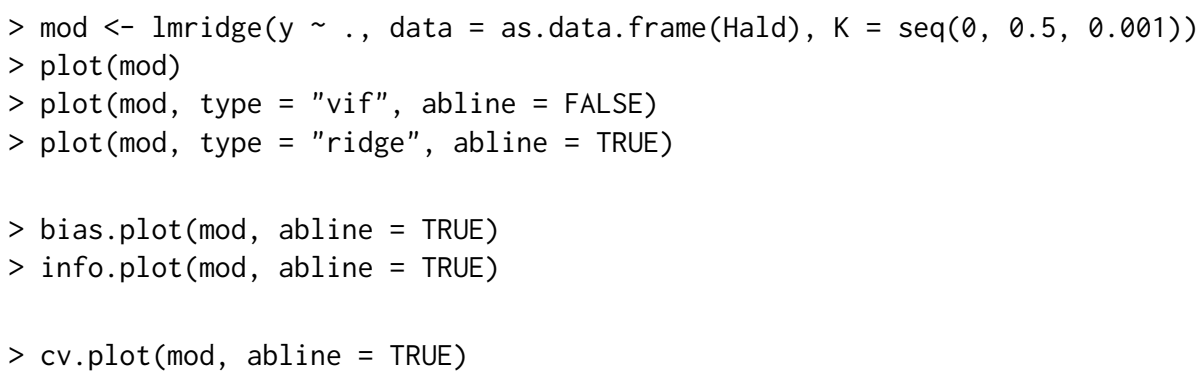

The vertical lines in ridge trace and VIF trace suggest the optimal value of biasing parameter $k$ selected at which GCV is minimum. The horizontal line in ridge trace is reference line at $y=0$ for ridge coefficient against vertical axis .

The bias-variance tradeoff plot (Figure 3) may be used to select optimal $k$ using bias.plot() function. The vertical line in bias-variance tradeoff plot shows the value of biasing parameter $k$ and horizontal line shows minimum MSE for ridge.

The plot of model selection criteria AIC and BIC for choosing optimal $k$ (Figure 4), info. plot() function may be used,

Function $\mathrm{cv}$. plot () plots the CV and GCV cross validation against biasing parameter $k$ for the optimal selection of $k$ (see Figure 5), that is, 


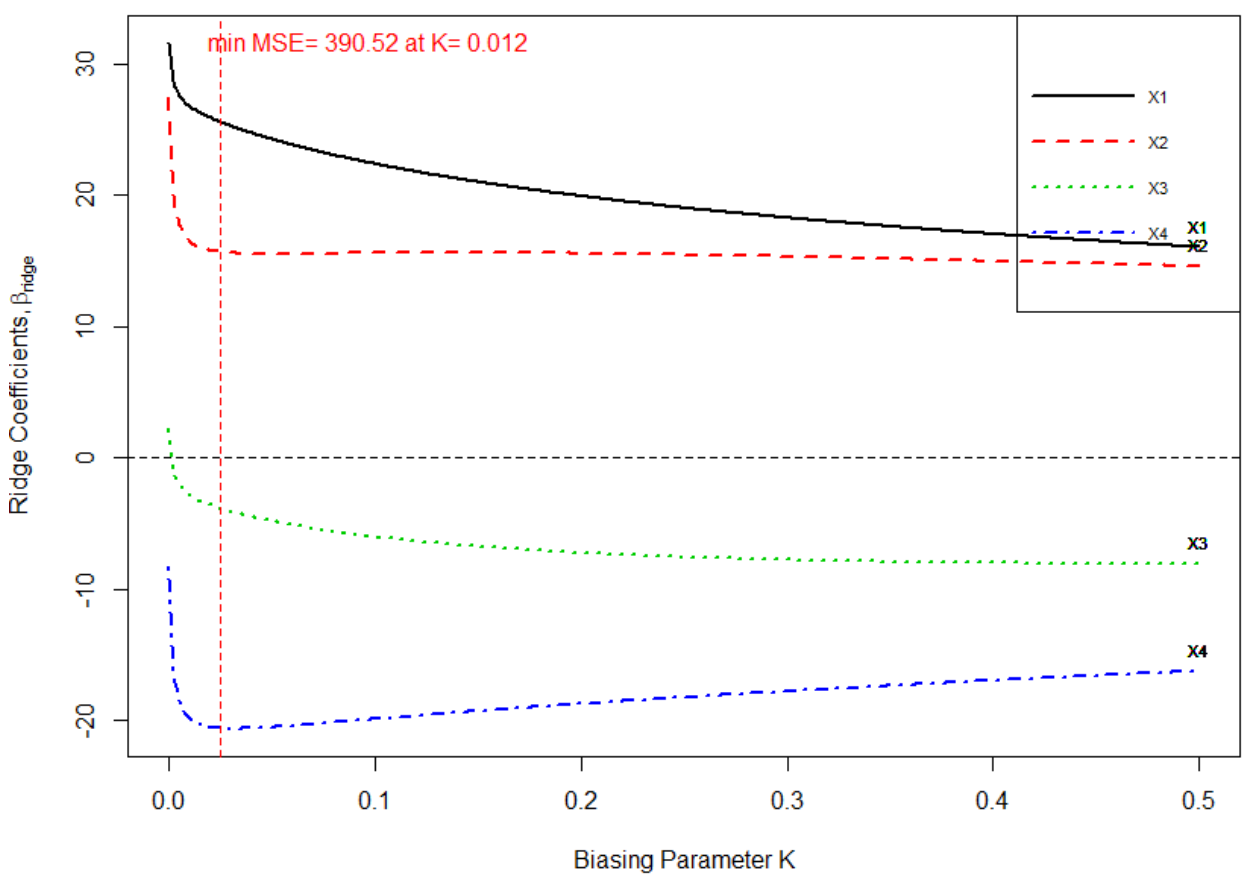

Figure 1: Ridge trace plot.

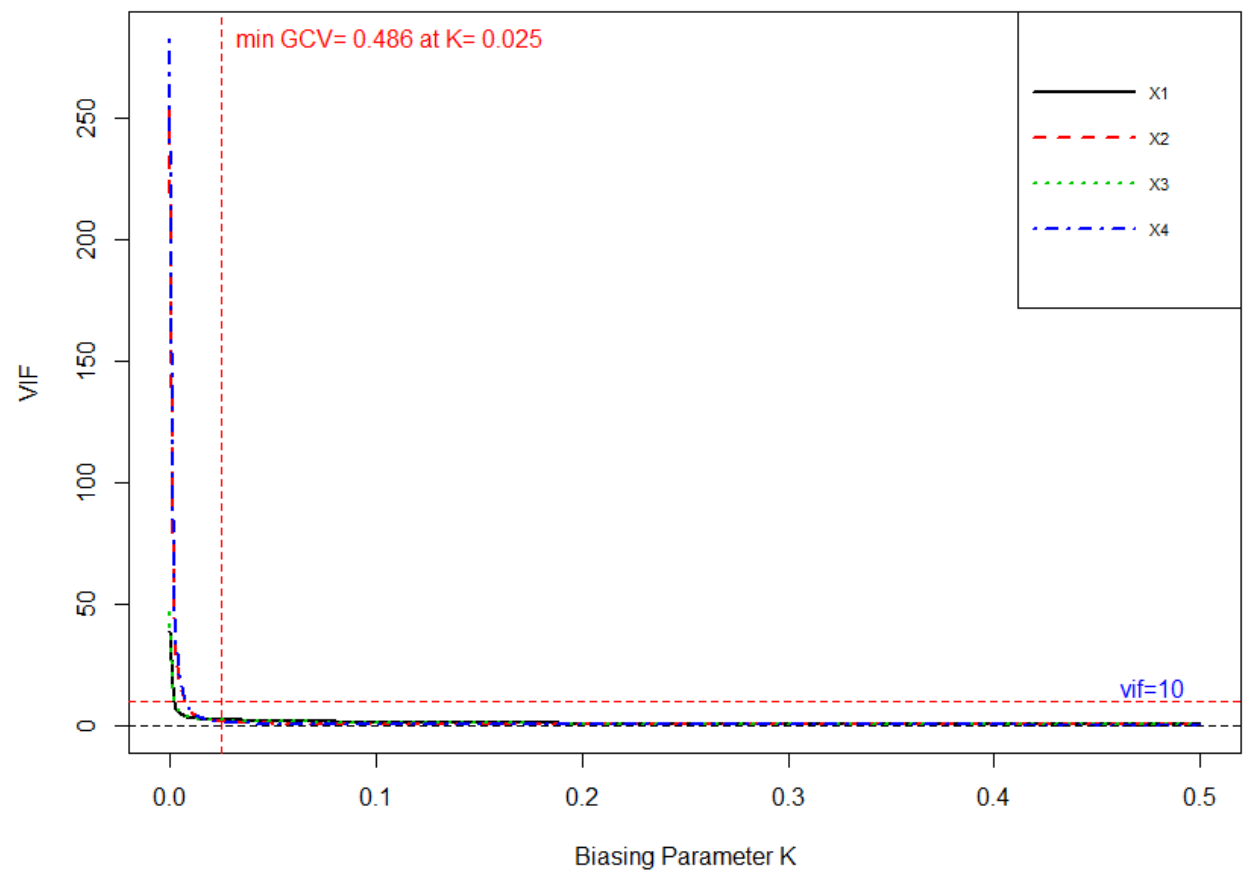

Figure 2: VIF trace.

$>$ isrm.plot(mod)

The $m$-scale and ISRM (Figure 6) measures by Vinod (1976) can also be plotted from function of isrm. plot() and can be used to judge the optimal value of $k$.

Function rplots.plot() plots the panel of three plots namely (i) df trace, (ii) RSS vs $k$ and (iii) PRESS vs $k$ and may be used to judge the optimal value of $k$, see Figure 7 .

$>$ rplots.plot(mod) 


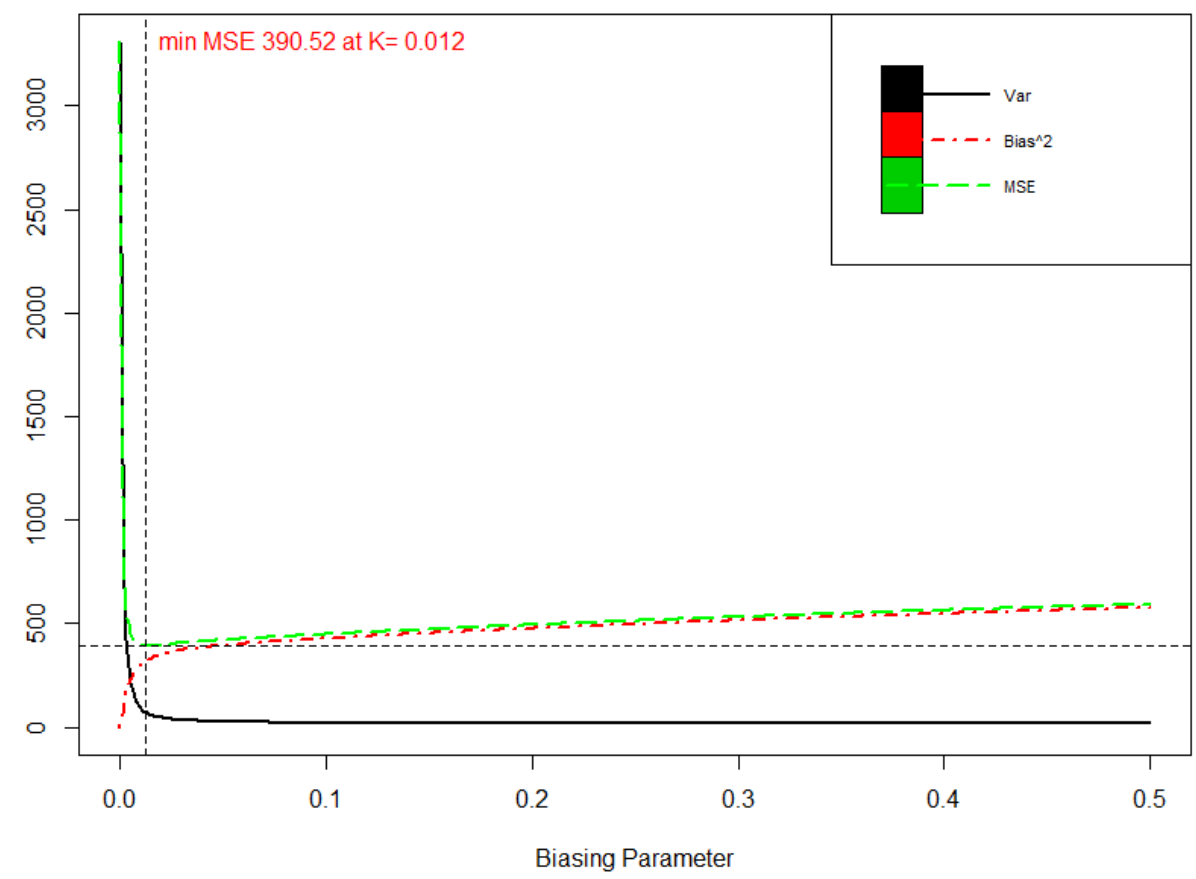

Figure 3: Bias-variance trade-off.

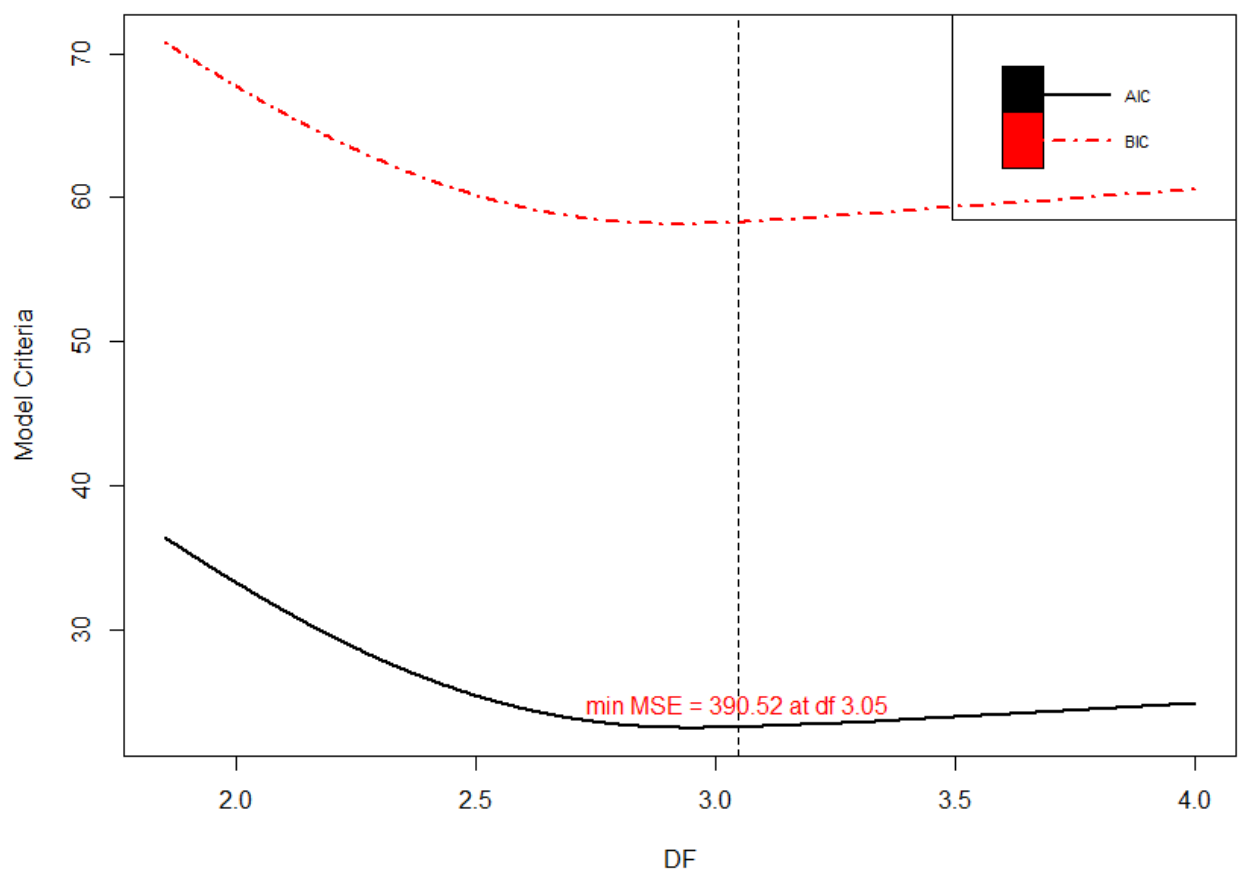

Figure 4: Information criteria plot (AIC and BIC).

\section{Summary}

Our developed lmridge package provides the most complete suite of tools for RR available in $\mathrm{R}$, comparable to those available as listed in Table 1. We have implemented functions to compute the ridge coefficients, testing of these coefficients, computation of different ridge related statistics and computation of the biasing parameter for different existing methods by various authors (see Table 4).

We have greatly increased the ridge related statistics and different graphical methods for the selection of biasing parameter $k$ through lmridge package in $\mathrm{R}$.

Up to now, a complete suite of tools for RR was not available for an open source or paid version of statistical software packages, resulting in reduced awareness and use of developed ridge related statistics. The package lmridge provides a complete open source suite of tools for the computation of 

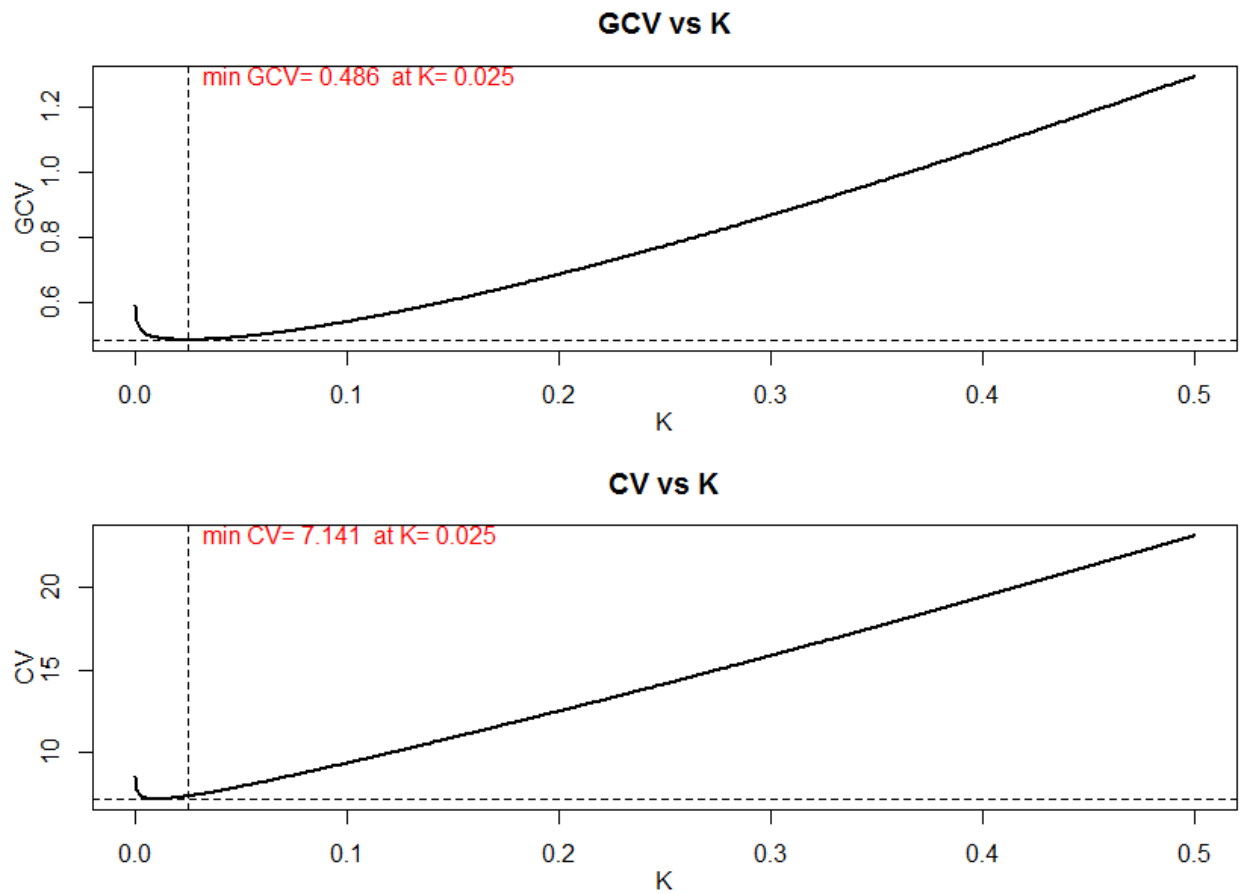

Figure 5: Cross-validation plots (CV and GCV).

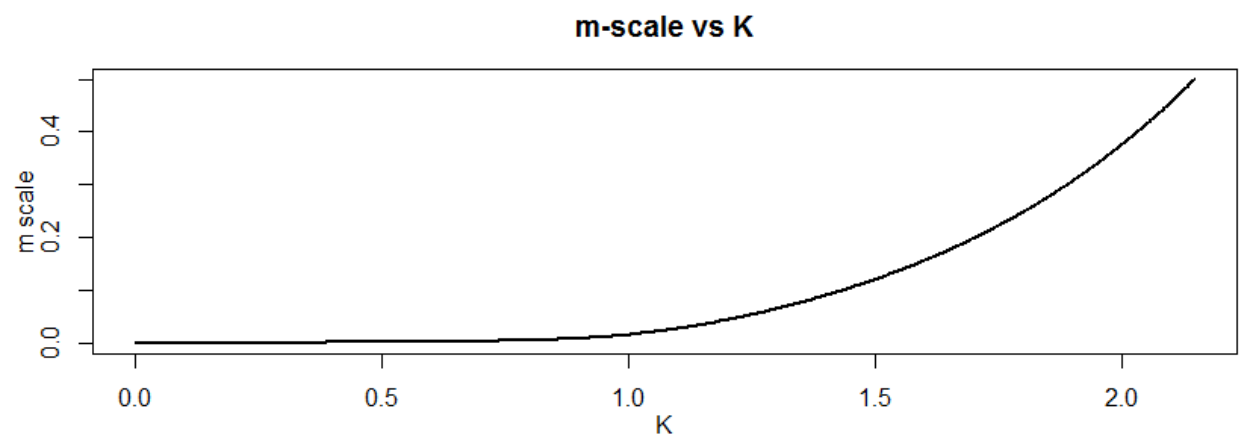

ISRM vs $\mathrm{K}$

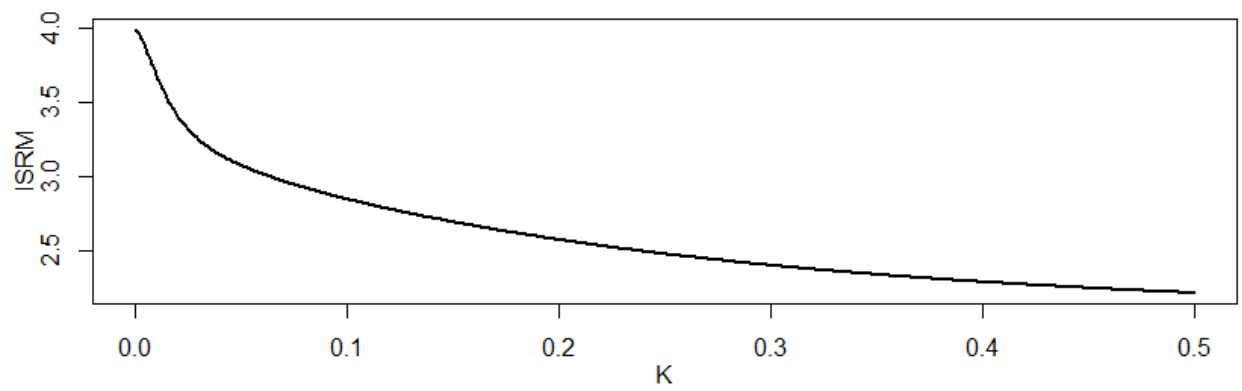

Figure 6: m-scale and ISRM plot.

ridge coefficients estimation, testing and computation of different statistics. We believe the availability of these tools will lead to increase utilization and better ridge related practices.

\section{Bibliography}

H. Akaike. A New Look at the Statistical Model Identification. IEEE Transaction on Automatic Control, 9 (6):716-723, 1973. URL https://doi.org/10.1109/TAC. 1974.1100705. [p332]

M. A. Alkhamisi and G. Shukur. A Monte Carlo Study of Recent Ridge Parameters. Communica- 

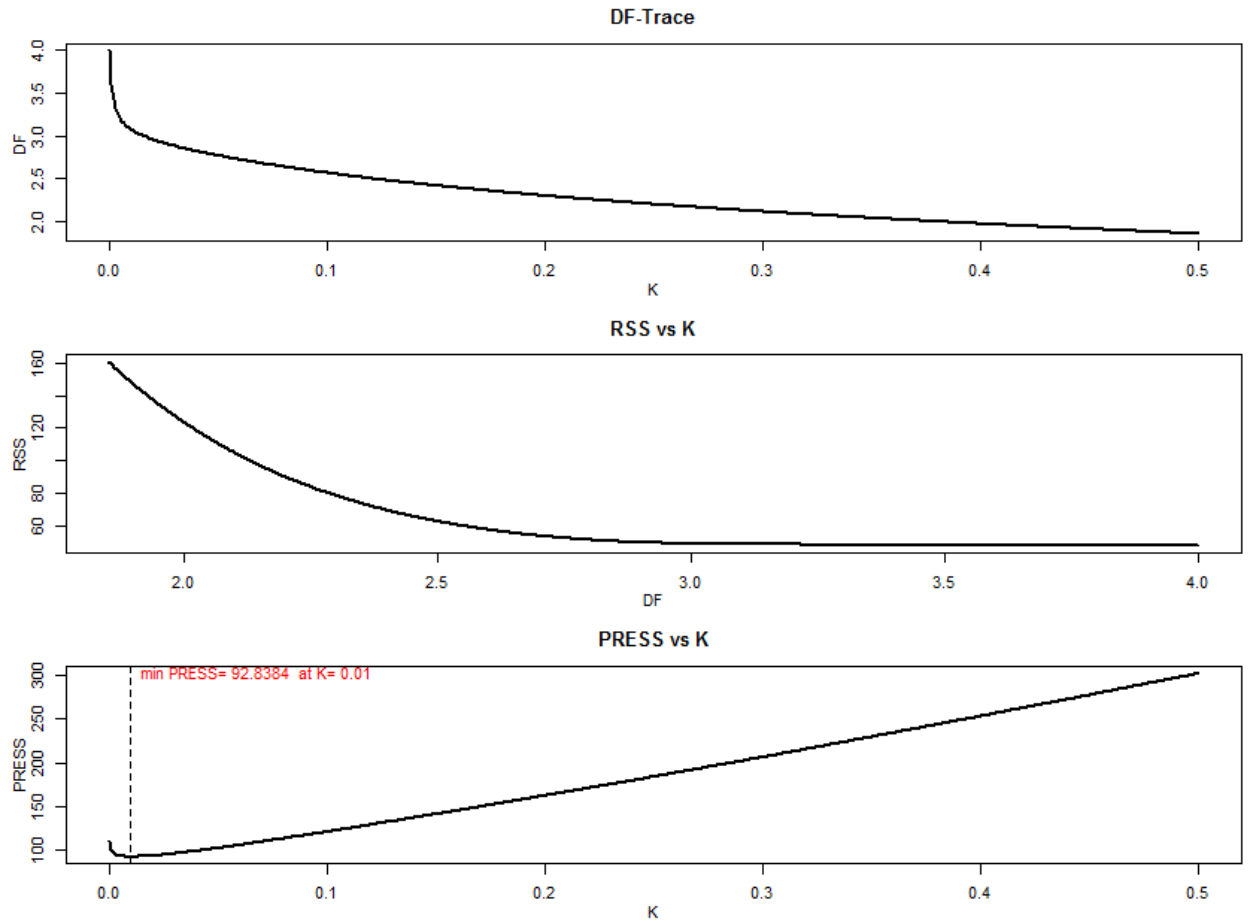

Figure 7: Miscellaneous ridge plots.

tions in Statistics-Simulation and Computation, 36(3):535-547, 2007. URL https: //doi .org/10.1080/ 03610910701208619. [p330]

M. A. Alkhamisi, G. Khalaf, and G. Shukur. Some Modifications for Choosing Ridge Parameter. Communications in Statistics-Theory and Methods, 35(11):2005-2020, 2006. URL https://doi .org/10. 1080/03610920600762905. [p330]

D. M. Allen. Mean Square Error of Prediction as a Criterion for Selecting Variables. Technometrics, 13 (3):469-475, 1971. URL http://doi .org/10.2307/1267161. [p332]

D. M. Allen. The Relationship Between Variable Selection and Data Augmentation and Method for Prediction. Technometrics, 16(1):125-127, 1974. URL http://doi .org/10.2307/1267500. [p330, 332]

S. Arumairajan and P. Wijekoon. Optimal generalized biased estimator in linear regression model. Open Journal of Statistics, 5:403-411, 2015. URL https://doi .org/10.4236/ojs. 2015. 55042. [p327]

M. Aslam. Using Heteroscedasticity-Consistent Standard Errors for the Linear Regression Model with Correlated Regressors. Communications in Statistics-Simulation and Computation, 43(10):2353-2373, 2014. URL https://doi.org/10.1080/03610918.2012.750354. [p332]

D. A. Belsley. A Guide to Using the Collinearity Diagnostics. Computer Science in Economics and Management, 4(1):33-50, 1991. URL https://doi .org/10.1007/BF00426854. [p334]

D. A. Belsley, E. Kuh, and R. E. Welsch. Diagnostics: Identifying Influential Data and Sources of Collinearity. John Wiley \& Sons, New York, 1980. chap. 3. [p328, 330]

S. Chatterjee and A. S. Hadi. Regression Analysis by Example. John Wiley \& Sons, 4th edition, 2006. [p328, 330]

E. Cule and M. De Iorio. A Semi-Automatic Method to Guide the Choice of Ridge Regression. Annals of Applied Statistics, arxiv:1205.0686v1, 2012. URL https://arxiv.org/abs/1205.0686v1. [p328, 334]

J. D. Curto and J. C. Pinto. The Corrected VIF (CVIF). Journal of Applied Statistics, 38(7):1499-1507, 2011. URL https://doi.org/10.1080/02664763.2010.505956. [p328]

N. J. Delaney and S. Chatterjee. Use of the Bootstrap and Cross-Validation in Ridge Regression. Journal of Business \& Economic Statistics, 4(2):255-262, 1986. URL http: //doi .org/10. 2307/1391324. [p332] 
A. Dissanayake, P. Wijekoon, and R-Core. Imrest: Different Types of Estimators to Deal with Multicollinearity, 2016. URL https://cran.r-project.org/package=lrmest. Rpackageversion3. 0 . [p327]

A. V. Dorugade. New Ridge Parameters for Ridge Regression. Journal of the Association of Arab Universities for Basic and Applied Sciences, 15:94-99, 2014. URL https://doi .org/10.1016/j . jaubas. 2013.03.005. [p333]

A. V. Dorugade and D. N. Kashid. Alternative Method for Choosing Ridge Parameter for Regression. Applied Mathematical Sciences, 4(9):447-456, 2010. [p333]

N. R. Draper and H. Smith. Applied Regression Analysis. John Wiley \& Sons, New York, 2nd edition, 1998. [p330, 334]

T. D. Dwividi and V. K. Shrivastava. On the Mimimum Mean Square Error Estimators in a Regression Model. Communications in Statistics-Theory and Methods, 7(5):487-494, 1978. URL https: //doi .org/ 10.1080/03610927808827642. [p333]

J. B. Endelman. Ridge Regression and Other Kernels for Genomic Selection with R Package rrBLUP. Plant Genome, 4(3):250-255, 2011. URL http://doi .org/10.3835/plantgenome2011.08.0024. [p328]

D. E. Farrar and R. R. Glauber. Multicollinearity in Regression Analysis: The Problem Revisted. The Review of Economics and Statistics, 49(1):92-107, 1967. URL http://doi . org/10. 2307/1937887. [p328]

J. Fox and S. Weisberg. An R Companion to Applied Regression. Sage, Thousand Oaks, CA, 2nd edition, 2011. URL https://socialsciences.mcmaster.ca/jfox/Books/Companion. [p328]

J. Friedman, T. Hastie, and R. Tibshirani. Regulaziation Paths for Generalized Linear Models via Coordinate Decent. Journal of Statistical Software, 33(1):1-22, 2010. URL http://doi .org/10.18637/ jss.v033.i01. [p328]

M. Friendly. genridge: Generalized Ridge Trace Plots for Ridge Regression, 2017. URL https: //cran.r-project.org/web/packages/genridge. R package version 0.6-6. [p328]

J. J. Goeman, R. J. Meijer, and N. Chaturvedi. Penalized: L1 (Lasso and Fused Lasso) and L2 (Ridge) Penalized Estimation in GLMs and in the Cox Model, 2017. URL https://cran.r-project.org/ web/packages/penalized. R package version 0.9-50. [p327]

G. H. Golub, M. Heath, and G. Wahba. Generalized Cross Validation as a Method for Choosing a Good Ridge Parameter. Technometrics, 21(2):215-223, 1979. URL http: //doi .org/10. 2307/1268518. [p328, 332]

W. H. Greene. Econometric Analysis. Prentic Hall, New Jersey, 5th edition, 2002. [p328]

D. K. Guilkey and J. L. Murphy. Direct Ridge Regression Techniques in Cases of Multicollinearity. Journal of American Statistical Association, 70(352):769-775, 1975. URL http://doi .org/10.1080/ 01621459.1975.10480301. [p330]

R. F. Gunst and R. L. Mason. Advantages of Examining Multicollinearities in Regression Analysis. Biometrics, 33(1):249-260, 1977. [p328]

W. Hadley. R Packages: Organize, Test, Document, and Share Your Code. O’Reilly Media, 2015. [p326]

A. M. Halawa and M. Y. El-Bassiouni. Tests of Regression Coefficients Under Ridge Regression Models. Journal of Statistical-Computation and Simulation, 65(1-4):341-356, 2000. URL https://doi . org/10. 1080/00949650008812006. [p332, 334]

A. Hald. Statistical Theory with Engineering Applications. John Wiley \& Sons, 1952. [p328]

T. Hastie and R. Tibshirani. Generalized Additive Models. Chapman \& Hall, 1990. [p334, 337]

W. J. Hemmerle. An Explicit Solution for Generalized Ridge Regression. Technometrics, 17(3):309-314, 1975. URL http://doi .org/10.2307/1268066. [p330]

W. J. Hemmerle and T. F. Brantle. Explicit and Constrained Generalized Ridge Estimation. Technometrics, 20(2):109-120, 1978. URL http://doi .org/10.2307/1268701. [p330]

R. R. Hocking, F. M. Speed, and M. J. Lynn. A Class of Biased Estimators in Linear Regression. Technometrics, 18(4):425-437, 1976. URL http://doi.org/10.2307/1268658. [p330] 
A. E. Hoerl. Optimum Solution of Many Variables Equations. Chemical Engineering Progress, 55:67-78, 1959. [p329]

A. E. Hoerl. Application of Ridge Analysis to Regression Problems. Chemical Engineering Progress, 58: 54-59, 1962. URL http://doi.org/10.1002/sim. 4780030311. [p329]

A. E. Hoerl. Ridge Analysis. Chemical Engineering Progress Symposium Series, 60:67-77, 1964. [p329]

A. E. Hoerl and R. W. Kennard. Ridge Regression: Biased Estimation of Nonorthogonal Problems. Technometrics, 12(1):55-67, 1970a. URL http://doi .org/10.2307/1267351. [p326, 328, 329, 330, 333]

A. E. Hoerl and R. W. Kennard. Ridge Regression: Application to Nonorthogonal Problems. Technometrics, 12(1):69-82, 1970b. URL http://doi .org/10.2307/1267352. [p329, 330]

A. E. Hoerl, R. W. Kennard, and K. F. Baldwin. Ridge Regression: Some Simulations. Communications in Statistics, 4(2):105-123, 1975. URL https: //doi .org/10.1080/03610927508827232. [p328]

M. U. Imdad and M. Aslam. mctest: An R Package for Detection of Collinearity Among Regressors, 2018a. URL https://cran.r-project.org/web/packages/mctest/. R package version 1.2. [p328]

M. U. Imdad and M. Aslam. lmridge: Linear Ridge Regression with Ridge Penalty and Ridge Statistics, 2018b. URL https://cran. r-project.org/package=lmridge. R package version 1.2. [p326]

M. Imdadullah, M. Aslam, and S. Altaf. mctest: An R Package for Detection of Collinearity Among Regressors. The R Journal, 8(2):495-505, 2016. URL https://journal.r-project.org/archive/ accepted/imdadullah-aslam-altaf.pdf. [p328]

S. I. Inc. The SAS System for Windows: Release 9.2, 2011. SAS Inst., Cary, NC. [p326]

B. Kan-Kilinc and O. Alpu. ltsbase: Ridge and Liu Estimates Based on LTS (Least Trimmed Squares) Method, 2013. URL https://CRAN.R-project.org/package=ltsbase. R package version 1.0.1. [p327]

B. Kan-Kilinc and O. Alpu. Combining Some Biased Estimation Methods with Least Trimmed Squares Regression and Its Application. Revista Colombiana de Estadística, 38(2):485-502, 2015. URL http://dx.doi.org/10.15446/rce.v38n2.51675. [p327]

J. D. Kasarda and W. F. Shih. Optimal Bias in Ridge Regression Approaches to Multicollinearity. Sociologial Methods and Research, 5(4):461-470, 1977. URL https://doi .org/10.1177/004912417700500405. [p330]

M. G. Kendall. A Course in Multivariate Analysis. Griffin, London, 1957. pp. 70-75. [p328]

R. W. Kennard. A Note on the Cp-Statistics. Technometrics, 13(4):899-900, 1971. URL https://doi . org/10.1080/00401706.1971.10488863. [p332]

G. Khalaf. A Comparison Between Biased and Unbiased Estimators. Journal of Modern Applied Statistical Methods, 12(2):293-303, 2013. URL http://10.22237/jmasm/1383279360. [p330]

G. Khalaf and G. Shukur. Choosing Ridge Parameter for Regression Problems. Communications in Statistics-Theory and Methods, 34(5):1177-1182, 2005. URL https://doi.org/10.1081/STA200056836. [p330]

B. M. G. Kibria. Performance of Some New Ridge Regression Estimators. Communications in StatisticsSimulation and Computation, 32(2):419-435, 2003. URL https://doi .org/10.1081/SAC-120017499. [p330, 333]

L. R. Klein. An Introduction to Econometrics. Prentic Hall, Englewood, Cliffs, N. J., 1962. pp. 101. [p328]

J. Kmenta. Elements of Econometrics. Macmillan Publishing Company, New York, 2nd edition, 1980. pp. 431. [p326]

A. Koutsoyiannis. Theory of Econometrics. Macmillan Education Limited, 1977. [p328]

P. Kovács, T. Petres, and Tóth. A New Measure of Multicollinearity in Linear Regression Models. International Statistical Review / Revue Internationale de Statistique, 73(3):405-412, 2005. URL http: //doi.org/10.1111/j.1751-5823.2005. tb00156.x. [p328]

J. F. Lawless and P. Wang. A Simulation Study of Ridge and Other Regression Estimators. Communications in Statistics-Theory and Methods, 5(4):307-323, 1976. URL https://doi .org/10.1080/ 03610927608827353. [p330, 333] 
W. F. Lee. Model Estimation Using Ridge Regression with the Variance Normalization Criterion. Master thesis, Department of Educational Foundation, Memorial University of Newfoundland, 1979. [p332]

F. Leisch. Creating R Packages: A Tutorial. Compstat 2008-Proceedings in Computational Statistics, Physica Verlage, Heidelberg, Germay, 2008, 2008. URL ftp://cran.r-project.org/pub/R/doc/ contrib/Leisch-CreatingPackages.pdf. [p326]

G. S. Maddala. Introduction to Econometrics. Macmillan Publishing Company, New York, 1988. [p328]

C. L. Mallows. Some Comments on Cp. Technometrics, 15(4):661-675, 1973. URL http: //doi . org/10. 2307/1267380. [p332]

D. W. Marquardt. Generalized Inverses, Ridge Regression, Biased Linear Estimation, and Nonlinear Estimation. Technometrics, 12(3):591-612, 1970. URL http://doi .org/10.2307/1267205. [p328, 330]

B. T. McCallum. Artificial Orthogonalization in Regression Analysis. Review of Economics and Statistics, 52(1):110-113, 1970. URL http://doi .org/10.2307/1927606. [p330]

G. C. McDonald and D. I. Galarneau. A Monte Carlo Evaluation of Some Ridge-Type Estimators. Journal of the American Statistical Association, 70(350):407-416, 1975. URL http://doi . org/10 .2307/2285832. [p330]

D. C. Montgomery and E. A. Peck. Introduction to Linear Regression Analysis. John Wiley \& Sons, New York, 1982. [p326]

S. Moritz and E. Cule. ridge: Ridge Regression with Automatic Selection of the Penalty Parameter, 2017. URL https://cran.r-project.org/web/packages/ridge. R package version 2.2. [p326]

G. Muniz and B. M. G. Kibria. On Some Ridge Regression Estimators: An Empirical Comparisons. Communications in Statistics-Simulation and Computation, 38(3):621-630, 2009. URL https: //doi .org/ 10.1080/03610910802592838. [p333]

G. Muniz, B. M. G. Kibria, K. Mansson, and G. Shukur. On Developing Ridge Regression Parameters: A Graphical Investigation. Statistics and Operations Research Transactions, 36(2):115-138, 2012. [p333]

R. H. Myers. Classical and Modern Regression with Application. PWS-KENT Publishing Company, 2 edition, 1986. [p326]

NCSS 11 Statistical Software. Ridge Regression, 2016. URL http://ncss . wpengine. netdna-cdn. com/ wp-content/themes/ncss/pdf/Procedures/NCSS/Ridge_Regression.pdf. NCSS, LLC. Kaysville, Utah, USA. [p326]

J. P. Newhouse and S. D. Oman. An Evaluation of Ridge Estimators. Rand Report, R-716-PR, 1971. [p330]

L. Nordberg. A Procedure for Determination of a Good Ridge Parameter in Linear Regression. Communications in Statistics-Simulation and Computation, 11(3):285-309, 1982. URL https: //doi .org/ 10.1080/03610918208812264. [p330]

B. Obenchain. RXshrink: Maximum Likelihood Shrinkage via Generalized Ridge or Least Angle Regression, 2014. URL https://cran.r-project.org/web/packages/RXshrink. R package version 1.0-8. [p328]

R. L. Obenchain. Classical F-Tests and Confidence Regions for Ridge Regression. Technometrics, 19(4): 429-439, 1977. URL http://doi.org/10.2307/1267882. [p330, 332]

A. Perperoglou. CoxRidge: Cox Models with Dynamic Ridge Penalties, 2015. URL https://cran . rproject.org/web/packages/CoxRidge. R package version 0.9.2. [p328]

B. S. Price. RidgeFusion: R Package for Ridge Fusion in Statistical Learning, 2014. URL https: //cran.r-project.org/web/packages/RidgeFusion. R package version 1.0-3. [p328]

R Core Team. Writing R Extensions. R Foundation for Statistical Computing, 2015. Version R 3.2.3. [p326]

J. O. Rawlings, S. G. Pantula, and D. A. Dickey. Applied Regression Analysis: A Research Tool. SpringerVerlag, New York, 2nd edition, 1998. [p326]

S-PLUS. Insightful Corporation, Seattle, Wt., 2008. URL http: //www. insightful. com. [p328] 
A. K. M. E. Saleh and B. M. G. Kibria. Peformance of Some New Preliminary Test Ridge Regression Estimators and Their Properties. Communications in Statistics-Theory and Methods, 22(10):2747-2764, 1993. URL https://doi.org/10.1080/03610929308831183. [p330]

G. E. Schwarz. Estimating the Dimension of a Model. The Annals of Statistics, 6(2):461-464, 1978. URL https://doi.org/10.1214/aos/1176344136. [p332]

G. A. F. Seber and A. J. Lee. Linear Regression Analysis. John Wiley \& Sons, New Jersey, 2 edition, 2003. [p326, 329]

B. Seifert. lpridge: Local Polynomial (Ridge) Regression, 2013. URL https: //cran.r-project/web/ packages/lpridge. R package version 1.0-7. [p328]

Shazam. Reference Manual Version 11, 2011. ISBN: 978-0-9570475-0-1. [p328]

X. Shen, M. Alam, F. Fikse, and L. Rönnegård. A Novel Generalized Ridge Regression Method for Quantitative Genetics. Genetics, 193(4):1255-1268, 2013. URL http://doi . org/10.1534/genetics . 112.146720. [p328]

S. Singh and D. S. Tracy. Ridge-Regression Using Scrambled Responses. Metrika, LVII(1-2):147-157, 1999. [p330]

StataCorp. Stata Statistical Software: Release 8, 2014. URL http://www. stata.com. College Station, TX: StataCorp LP. [p327]

H. Theil. Specification Errors and the Estimation of Economic Relationships. International Statistical Institute (ISI), 25(1/3):41-51, 1957. URL http://doi.org/10.2307/1401673. [p329]

H. Theil. Principles of Econometrics. John Wiley \& Sons, New York, 1971. [p328]

R. A. Thisted. Ridge Regression, Minimax Estimation, and Empirical Bayes Methods. Technical Report 28, Stanford, Calif.: Division of Biostatistics, Stanford University, 1976. [p333]

R. E. Tripp. Non-Stochastic Ridge Regression and Effective Rank of the Regressors Matrix. Ph.d. thesis, Department of Statistic, Virginia Polytechnic Institute and State University., 1983. [p326]

W. N. Venables and B. D. Ripley. Modern Applied Statistics with S. Springer-Verlag, New York, 4th edition, 2002. URL http: // www. stats.ox. ac.uk/pub/MASS4. ISBN 0-387-95457-0. [p326, 328, 333]

H. D. Vinod. Application of New Ridge Regression Methods to a Study of Bell System Scale Economics. Journal of the American Statistical Association, 71(356):835-841, 1976. URL http://doi . org/10.2307/ 2286847. [p330, 332, 339]

E. Wencheko. Estimation of the Signal-to-Noise in the Linear Regression Model. Statistical Papers, 41: 327-343, 2000. URL https://doi .org/10.1007/BF02925926. [p330]

D. W. Wichern and G. A. Churchill. A Comparison of Ridge Estimators. Technometrics, 20(3):301-311, 1978. URL http://doi.org/10.2307/1268139. [p330]

Muhammad Imdad Ullah

Ph.D scholar (Statistics),

Department of Statistics

Bahauddin Zakariya University, Multan, Pakistan

ORCiD ID: 0000-0002-1315-491X

mimdadasad@gmail.com

Muhammad Aslam

Associate Professor,

Department of Statistics

Bahauddin Zakariya University, Multan, Pakistan

aslamasadi@bzu.edu.pk

Saima Altaf

Assistant Professor,

Department of Statistics

Bahaudding Zakariya University, Multan, Pakistan

saimaaltaf@bzu.edu.pk 